\title{
A Time Discretization Scheme for a Nonlocal Degenerate Problem Modelling Resistance Spot Welding
}

\author{
N. I. Kavallaris *, Y. Yan \\ ${ }^{1}$ Department of Mathematics, University of Chester Thornton Science Park \\ Pool Lane, Ince, Chester CH2 4NU, UK
}

\begin{abstract}
In the current work we construct a nonlocal mathematical model describing the phase transition occurs during the resistance spot welding process in the industry of metallurgy. We then consider a time discretization scheme for solving the resulting nonlocal moving boundary problem. The scheme consists of solving at each time step a linear elliptic partial differential equation and then making a correction to account for the nonlinearity. The stability and error estimates of the developed scheme are investigated. Finally some numerical results are presented confirming the efficiency of the developed numerical algorithm.
\end{abstract}

Keywords and phrases: Non-local, degenerate parabolic equation, moving boundary, stability, error estimates, Chernoff formula, resistance spot welding

Mathematics Subject Classification: Primary 65N15, 65N30, Secondary 35K55, $35 \mathrm{~K} 65,35 \mathrm{R} 35$

\section{Introduction}

In the current work we consider the following nonlocal degenerate problem

$$
\begin{aligned}
& \frac{\partial u}{\partial t}=\Delta \beta(u)+\frac{\lambda f(\beta(u))}{\left(\int_{\Omega} f(\beta(u)) d x\right)^{p}}, \text { in } Q_{T}:=\Omega \times(0, T), T>0, \\
& \beta(u)+k(x) \frac{\partial \beta(u)}{\partial \mathbf{n}}=0, \quad \text { on } \partial \Omega \times(0, T), \\
& u(x, 0)=u_{0}(x), \quad \text { in } \Omega,
\end{aligned}
$$

where $\lambda, p$ are positive constants and $\frac{\partial}{\partial \mathbf{n}}=\nabla \cdot \mathbf{n}$ denotes the normal derivative on the boundary $\Gamma=\partial \Omega$.

The characterization of (1.1) as nonlocal is due to existence of the integral appearing in the denominator of the source term. Here $\beta(u)$ stands for a continuous function defined on $\mathbb{R}$ satisfying $\beta(0)=0$ and $\Omega$ is a polyhedral and convex domain in $\mathbb{R}^{d}(d \geq 1)$. A common case for function $\beta$ is the power-law function $\beta(u)=u^{m}, m>0$, and then (1.1) is a nonlocal porous medium equation. Nevertheless, under the general condition $\beta^{\prime}(u) \geq 0$, which is actually necessary for making equation (1.1) formally parabolic, equation (1.1) is called a nonlocal filtration equation. The nonlinear function $f(s)$ can be considered, for

*Corresponding author. E-mail: n.kavallaris@chester.ac.uk, y.yan@chester.ac.uk

(C) EDP Sciences, 2015 
some applications, as monotonic though in the current paper we only assume to be positive and Lipschitz continuous. Also $k(x)$ is considered in $C^{1+\delta}(\Gamma), \delta>0$, and $k(x)=0, k(x)=\infty$ and $0<k(x)<\infty$ correspond to Dirichlet, Neumann and Robin (mixed) type boundary conditions respectively.

Since $f(s)>0$, whenever $u_{0}(x)>0$ in $\Omega$, we obtain via the comparison principle for the (local) porous medium operator that $u(x, t)>0$ in $\Omega \times(0, T)$ and hence problem (1.1) - (1.3) is non-degenerate, [22]. Consequently local-in-time existence and uniqueness of a classical solution to (1.1) - (1.3) is guaranteed and can be obtained by classical arguments, $[8,13]$. Furthermore long-time behaviour was studied by using comparison and energy methods for different nonlinear functions $f(s)$, [8,13,20,21]. Even finitetime and infinite blow-up of the solution of (1.1) - (1.3) is proven to occur under some circumstances in the non-degenerate case, see $[8,10,13,14,20,21]$. Consequently local-in-time existence and uniqueness of a classical solution to (1.1) - (1.3) is guaranteed and can be obtained by classical arguments, $[8,13]$. Furthermore long-time behaviour was studied by using comparison and energy methods for different nonlinear functions $f(s),[8,13,20,21]$. Even finite-time and infinite blow-up of the solution of (1.1) (1.3) is proven to occur under some circumstances in the non-degenerate case, see $[8,10,13,14,20,21]$.

On the other hand, when $u_{0}(x)$ has compact support i.e. there exists a bounded closed set $\Omega_{0} \varsubsetneqq \Omega$ such that $u_{0}(x)=0$, for $x \notin \Omega_{0}$ then $u(x, t)$ will remain compactly supported for all later times $t$, thus problem (1.1) - (1.3) becomes degenerate. In that case only a weak solution of (1.1) - (1.3) can be defined which is a function $u \in L^{2}\left(Q_{T}\right)$ with $\beta(u) \in L^{\infty}\left((0, T) ; L^{2}(\Omega)\right) \cap L^{2}\left((0, T) ; H_{0}^{1}(\Omega)\right)$ and

$$
\frac{\lambda f(\beta(u))}{\left(\int_{\Omega} f(\beta(u)) d x\right)^{p}} \in L^{2}\left(Q_{T}\right)
$$

which satisfies the equation

$$
\begin{aligned}
& \int_{\Omega}\left[u\left(x, t_{1}\right) v\left(x, t_{1}\right)-u(x, 0) v(x, 0)\right] d x \\
& =\int_{0}^{t_{1}} \int_{\Omega}\left(u v_{t}-\nabla \beta(u) \cdot \nabla v+\frac{\lambda f(\beta(u)) v}{\left(\int_{\Omega} f(\beta(u)) d x\right)^{p}}\right) d x d t
\end{aligned}
$$

for any $t_{1} \in[0, T]$ and $v \in \dot{H}^{1,1}\left(Q_{t_{1}}\right)$ (where $\dot{H}^{1,1}\left(Q_{t_{1}}\right)$ is the closure of $H^{1,1}\left(Q_{t_{1}}\right)$ with respect to its norm) with $v=0$ on $\partial \Omega$, [8].

This degeneracy results into the interesting phenomenon of the finite speed of propagation. Indeed, a moving boundary is formed, called also interface, separating the regions $P_{u}(t)=\{x \in \Omega: \beta(u(x, t))>0\}$ and $N_{u}(t)=\{x \in \Omega: \beta(u(x, t))<0\}$. It is defined as $\Gamma_{u}(t)=\partial P_{u} \cap Q_{T}$ and propagates with finite speed. A intriguing problem both from mathematical and application point of view is the determination of the evolution in time of the moving boundary. In the current paper we develop a numerical scheme which supplies an approximation of the moving boundary and also provides a uniform approach for both non-degenerate and degenerate cases.

The paper is organized as follows. In Section 2 we explain how the mathematical model (1.1)-(1.3) can describe the resistance spot welding process arising in metallurgy. In Section 3, we present the discretization scheme employed to approximate the solution of (1.1)-(1.3). The stability of this numerical scheme is investigated in Section 4. In Section 5, we provide the error estimates of the time discretization scheme (3.10)-(3.12). Section 6 is devoted to the presentation of some numerical experiments verifying the efficiency of the numerical scheme.

\section{The Mathematical Model}

Our original motivation for studying problem (1.1) - (1.3) comes from the so called resistance spot welding process. Resistance welding is a thermo-electric process in which Joule heating is generated at the interface of the parts to be joined by passing an electrical current through the parts for a precisely 


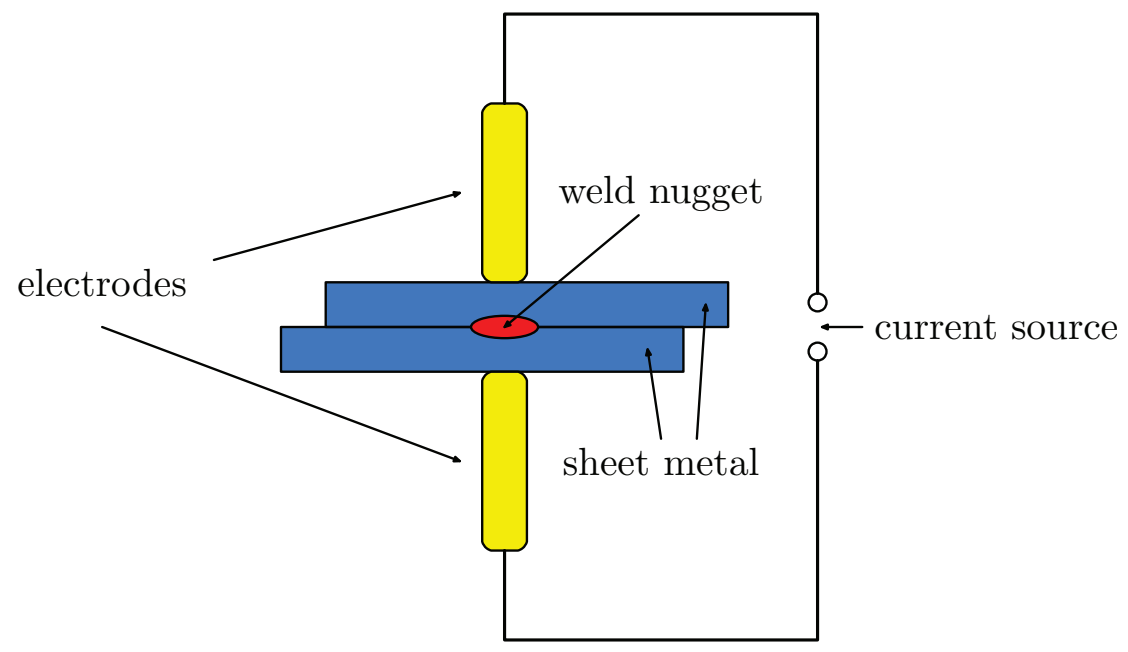

FiguRE 1. Schematic diagram of resistance spot welding system

controlled time and under a controlled pressure. The term "resistance" welding derives from the fact that the resistance of the workpieces and electrodes are used in combination or contrast to generate the heat at their interface. A common configuration of the resistance spot welding process can be seen in Figure 1. Key advantages of the resistance welding process include: very short process time, no consumables, operator safety because of low voltage, clean and environmentally friendly and finally a reliable electromechanical joint is formed. Resistance spot welding is the most popular joining process in automobile body assembly production lines; other applications include robotics, orthodontist's clinic and batteries manufacturing.

When an electric current, with local current density $\vec{j}$, flows through the electrodes and the sheet metals, then owning to a significantly higher resistivity in the contact area a rapid heating up of this area occurs, which is caused by the Joule effect. The rapid heating leads to the development of a weld nugget (a mushy region where the solid and liquid phases coexist) which actually grows quite fast. Once, the electrical current is switched off the weld nugget solidifies, leading to a lasting weld joint (known also as weldment) between the metal sheets. The main physical quantities are involved in the description of the configuration of Figure 1 are: the temperature $u$, a parameter $\psi$ standing for the proportion of the two phases and the applied electrical potential $\phi$. In the melting-solidification process $\psi=0$ in the solid phase while in the liquid phase we have $\psi=1$.

The temperature evolution of the above system is governed by the following internal energy balance equation

$$
\frac{\partial u}{\partial t}+\ell \frac{\partial \psi}{\partial t}=\nabla \cdot(k(u, \psi) \nabla u)+\rho(u, \psi)|\vec{j}|^{2} \quad \text { in } \quad Q_{T},
$$

where $k(u, \psi)$ and $\rho(u, \psi)$ represent the thermal conductivity and the electrical resistance of the metal sheets respectively whilst $\ell$ stands for the lateral heat of the phase change process. Here $\Omega$ denotes the joined part of the two metal sheets indicated with blue color in Figure 1. In case the lateral heat $\ell$ is small, a rather realistic assumption, then (2.1) reduces to

$$
\frac{\partial u}{\partial t}=\nabla \cdot(k(u, \psi) \nabla u)+\rho(u, \psi)|\vec{j}|^{2} \quad \text { in } \quad Q_{T} .
$$

Owning to Ohm's law the current density satisfies

$$
\vec{j}=-\tau(u, \psi) \nabla \phi,
$$


where $\tau(u, \psi)=1 / \rho(u, \psi)$ is the electrical conductivity of the metal sheets and thus (2.2) reads

$$
\left.\frac{\partial u}{\partial t}=\nabla \cdot(k(u, \psi) \nabla u)+\tau(u, \psi)\right)|\nabla \phi|^{2}, \quad \text { in } \quad Q_{T} .
$$

Also due to conservation of charge $\nabla \cdot \vec{j}=0$ and hence we derive the second governing equation of our system

$$
\nabla \cdot(\tau(u, \psi) \nabla \phi)=0, \quad \text { in } \quad Q_{T} .
$$

Next we describe the derivation of the third mastering equation of our system which describes the phase transition. It is usually caled the phase equation and describes the time-evolution of $0 \leq \psi \leq 1$. First we note that the free energy of the system can be chosen having the Landau-Ginzburg form

$$
F(u, \psi)=c_{V} u(1-\log u)+u\left(\hat{g}(\psi)+\mu \frac{|\nabla \psi|^{2}}{2}\right)+\ell \psi,
$$

where $c_{V}$ is the specific heat, taking henceforth equal to 1 for simplicity, and $\mu$ is a positive constant; $\hat{g}$ has usually the form of a double well potential, e.g.

$$
\hat{g}(\psi)=\frac{1}{2}\left(1-\psi^{2}\right)^{2},
$$

see $[5,7]$.

Assuming that our isothermal system moves towards local minima of the total free energy

$$
\mathcal{F}(u, \psi)=\int_{\Omega} F(u, \psi) d x,
$$

we can impose that the order-parameter dynamics is given by

$$
u \frac{\partial \psi}{\partial t}=-\delta_{\psi} \mathcal{F}(u, \psi), \quad \text { in } \quad Q_{T},
$$

where $\delta_{\psi} \mathcal{F}(u, \psi)$ represents the variational derivative of $\mathcal{F}$ with respect to $\psi$. Taking into account $(2.6)$ as well as the fact that $0<\ell \ll 1$ then $(2.7)$ yields

$$
\frac{\partial \psi}{\partial t}=\mu \Delta \psi+g(\psi), \quad \text { in } \quad Q_{T},
$$

where $g$ is the derivative of $\hat{g},[7]$.

From experimental data we can observe that usually both thermal and electrical conductivities have a discontinuity in the melting point, [17]. Actually we can assume the mixture ansatz $k(u, \psi)=(1-$ $\psi) \widetilde{k}_{1}(u)+\psi \widetilde{k}_{2}(u)$ and $\tau(u, \psi)=(1-\psi) \widetilde{\tau}_{1}(u)+\psi \widetilde{\tau}_{2}(u)$ with possibly different $\widetilde{k}_{1}, \widetilde{k}_{2}$ and $\widetilde{\tau}_{1}, \widetilde{\tau}_{2}$, see $[7,18]$. Or alternatively we have

$$
k(u, \psi)=\widetilde{k}(u)= \begin{cases}k_{1}(u), & \text { if } u<u_{m} \\ k_{2}(u), & \text { if } u>u_{m}\end{cases}
$$

and

$$
\tau(u, \psi)=\widetilde{\tau}(u)= \begin{cases}\tau_{1}(u), & \text { if } u<u_{m}, \\ \tau_{2}(u), & \text { if } u>u_{m}\end{cases}
$$

for $k_{1}(s) \neq k_{2}(s)$ and $\tau_{1}(s) \neq \tau_{2}(s)$. Here $u_{m}$ stands for the melting temperature of the metal workpieces. 


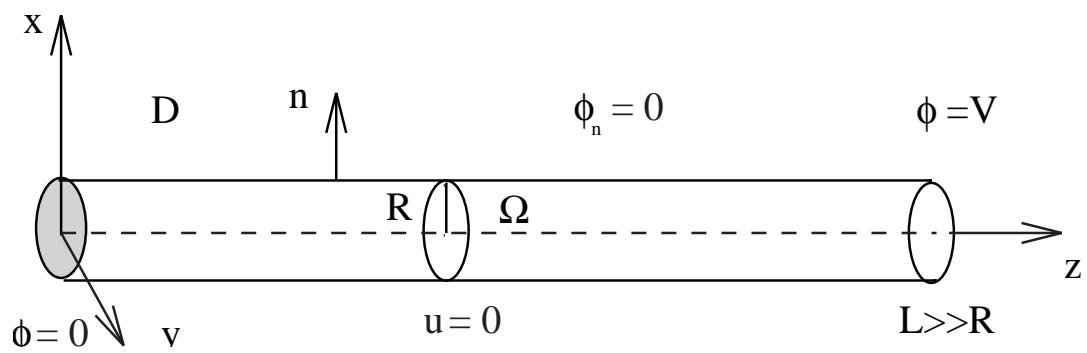

Figure 2. A long and thin cylindrical conduct area.

Then the melting-solidification process is described by

$$
\begin{aligned}
& \frac{\partial u}{\partial t}=\nabla \cdot(\widetilde{k}(u) \nabla u)+\widetilde{\tau}(u)|\nabla \phi|^{2} \text { in } Q_{T}, \\
& \nabla \cdot(\widetilde{\tau}(u) \nabla \phi)=0, \quad \text { in } \quad Q_{T} .
\end{aligned}
$$

Consider now the case where the contact area $\Omega$ is long and thin of length $L$ with axis parallel to $z$-direction and its ends at $z=0, L$ where $\phi=0, V$ respectively. Let $D$ be the cross section of the contact area $\Omega$, then we consider that its dimensions are much smaller compared $L$. For the case of a cylindrical contact area with diameter $R$ much less that the length $L$, i.e. $R \ll L$, see Figure 2 . We also assume that the conduct area $\Omega$ is electrically insulated, $\frac{\partial \phi}{\partial \mathbf{n}}=0$ on $\Gamma$, and the temperature is kept fixed on its curved surface, $u=0$ on $\Gamma$. Neglecting the end effects at $z=0, L$ we can actually take that the potential $\phi$ depends only on the $z$-variable and thus $(2.10)$ yields $\left(\widetilde{\tau}(u) \phi_{z}\right)_{z}=0$ which finally implies that $\left(\widetilde{\tau}(u) \phi_{z}\right)$ depends only on time $t$. Under integration over the cross section $D$ we take

$$
\int_{D} \widetilde{\tau}(u) \phi_{z} d x d y=\frac{I(t)}{A}
$$

where $I(t)$ is the total current flowing through each cross section of the conductor and $A=|D|$, thus

$$
\phi_{z}=\frac{I(t)}{A \int_{D} \widetilde{\tau}(u) d x d y} .
$$

Combining (2.9) and (2.11) and letting $x$ denotes the position in the cross-section and $\Delta$ the twodimensional Laplacian then system (2.9)-(2.10) is reduced to the single nonlocal equation

$$
\frac{\partial u}{\partial t}=\Delta K(u)+\frac{\lambda \widetilde{\tau}(u)}{\left(\int_{D} \widetilde{\tau}(u) d x\right)^{2}}, \quad \text { in } D \times(0, T)
$$

associated with boundary and initial conditions

$$
K(u)=0 \quad \text { on } \quad x \in \partial D,
$$

and

$$
u(x, 0)=u_{0}(x), x \in D,
$$

provided also that the total current $I(t)=I$ is constant. Here $\lambda=\frac{I^{2}}{A^{2}}$ is the parameter that actually controls the welding process. Also $K(u)$ is defined as

$$
K(u)=\int_{0}^{u} \widetilde{k}(s) d s .
$$


Alternatively, by applying Fourier's law for diffusion and heat conduction as well as conservation of energy, [13], we can take $u$ satisfying a mixed-type boundary condition

$$
K(u)+k(x) \frac{\partial K(u)}{\partial \mathbf{n}}=0 \quad \text { on } \quad x \in \partial D .
$$

To our knowledge the mathematical model (2.12) represents the first coupling of Joule effect and phasetransition under a single nonlocal equation.

We should point out here that a nonlocal model similar to (1.1) - (1.3) but for $p=1$ was introduced in [3] to describe adiabatic shear-banding formation phenomena in strained metals permitting phase transition in an isothermal manner as well. The mathematical model (1.1) - (1.3) can be also used when $p=2$ to describe the operation of thermistors in case thermal conductivity depends on temperature, [8], as well as modelling the phenomenon of flash sintering when also $\beta(u)=u$, [6]. Another nonlocal model of the form (1.1) - (1.3) for $\beta(u)=u$ and $p=1$ was used in $[11,24]$ to describe the biological phenomenon of chemotaxis under some special circumstances. Finally another nonlocal model arising in linear friction welding of metals is constructed and investigated in [9].

On the other hand, by using nonlinear diffusion profiles of the form $\beta(u)=u^{m}, m>0$, then (1.1) (1.3) is a proper model to describe the heat radiation produced by a plasma conductor (ionized gas) in high temperatures, see $[20,21,25]$, under the Joule heating effect. This is a very interesting application that will be treated in a forthcoming paper. The developed numerical algorithm in the current work cannot tackle such a case due to the failure of the Assumption $\left(H_{\beta}\right)$, thus a more sophisticated nonlinear numerical scheme is required.

\section{The numerical scheme}

Nochetto and Verdi [16] studied the following local nonlinear parabolic problem

$$
\begin{aligned}
& \frac{\partial u}{\partial t}-\Delta \beta(u)=f(\beta(u)), \quad \text { in } \Omega \times(0, T), \\
& \beta(u)=0, \quad \text { on } \partial \Omega \times(0, T), \\
& u(x, 0)=u_{0}(x), \quad \text { in } \Omega,
\end{aligned}
$$

where $\beta(s)$ satisfies the assumption $\left(H_{\beta}\right)$ below. They introduced a finite element method for solving (3.1) - (3.3) and the error estimates in both semidiscrete and fully discrete cases were derived. The usual technique to approximate (3.1) - (3.3) amounts to discretizing a nonlinear elliptic partial differential equations at each time step. The success of such a procedure relies on the smoothness of the solution $u$ and $\theta=\beta(u)$. It is not a priori obvious that standard techniques for mildly nonlinear parabolic equations apply on low-regularity (degenerate) problem (3.1) - (3.3). Based on this observation, Nochetto and Verdi [16] tried the following numerical scheme.

Let $0=t_{0}<t_{1}<\cdots<t_{N}=T$ be a partition of $[0, T]$. Let $U^{n} \approx u\left(t_{n}\right)$ and $\Theta^{n} \approx f\left(\beta\left(u\left(t_{n}\right)\right)\right)$ be the approximate solutions of $u\left(t_{n}\right)$ and $f\left(\beta\left(u\left(t_{n}\right)\right)\right)$. Nochetto and Verdi [16] introduced the following time discretization scheme which is a nonlinear Chernoff formula, find $\Theta^{n} \in H_{0}^{1}(\Omega), U^{n} \in L^{2}(\Omega)$, such that, for any $v \in H_{0}^{1}(\Omega)$,

$$
\begin{aligned}
& U^{0}:=u_{0}, \\
& \left(\Theta^{n}, v\right)-\frac{\tau}{\mu}\left(\nabla \Theta^{n}, \nabla v\right)=\left(\beta\left(U^{n-1}\right), v\right)+\frac{\tau}{\mu}\left(f\left(\beta\left(U^{n-1}\right)\right), v\right), \\
& U^{n}:=U^{n-1}+\mu\left(\Theta^{n}-\beta\left(U^{n-1}\right)\right), \quad 1 \leq n<N:=\frac{T}{\tau} .
\end{aligned}
$$

Actually the above scheme was first introduced in [4] for tackling the filtration equation $u_{t}=\Delta \beta(u)$. Here $\tau>0$ is the time step and $\mu>0$ is the relaxation parameter which satisfies the stability constraint 
$\mu<L_{\beta}^{-1}\left(L_{\beta}=\right.$ Lipschitz constant of $\left.\beta\right)$. The numerical scheme consists of solving at each time step a linear elliptic partial differential equation and then making a correction to account for the nonlinearity.

One of the first numerical studies on non-local problems of the form of (1.1) - (1.3) was done in [1]. The authors, in [1], considered the following nonlocal filtration problem

$$
\begin{aligned}
& \frac{\partial u}{\partial t}-\Delta \beta(u)=\frac{\lambda f(u)}{\left(\int_{\Omega} f(u) d x\right)^{2}}, \quad \text { in } \Omega \times(0, T), \\
& \beta(u)=0, \quad \text { on } \Gamma \times(0, T), \\
& u(x, 0)=u_{0}(x), \quad \text { in } \Omega .
\end{aligned}
$$

They introduced a time discretization scheme and proved the error estimates. However, no numerical results were presented in [1] for testing the validity and the efficiency of the produced numerical scheme.

In the current work, we introduce a similar to (3.4)-(3.6) time discretization scheme which captures all the key features of the possible degenerate problem (1.1)-(1.3) considering for simplicity only homogeneous Dirichlet boundary conditions. So we look for a $\Theta^{n} \in H_{0}^{1}(\Omega), U^{n} \in L^{2}(\Omega)$ such that, for any $v \in H_{0}^{1}(\Omega)$

$$
\begin{aligned}
& U^{0}:=u_{0}, \\
& \left(\Theta^{n}, v\right)-\frac{\tau}{\mu}\left(\nabla \Theta^{n}, \nabla v\right)=\left(\beta\left(U^{n-1}\right), v\right)+\frac{\lambda \tau}{\mu} \frac{\left(f\left(\beta\left(U^{n-1}\right)\right), v\right)}{\left(\int_{\Omega} f\left(\beta\left(U^{n-1}\right)\right) d x\right)^{2}}, \\
& U^{n}:=U^{n-1}+\mu\left(\Theta^{n}-\beta\left(U^{n-1}\right)\right), \quad 1 \leq n<N:=\frac{T}{\tau} .
\end{aligned}
$$

Denote $\theta(t)=\beta(u(t)), e_{\theta}=\theta(t)-\Theta^{n}$ and $e_{u}=u(t)-U^{n}$ for any $t \in\left(t_{n-1}, t_{n}\right)$, we will prove the following error estimates

$$
\left\|e_{\theta}\right\|_{L^{2}(\Omega \times(0, T))}+\left\|\int_{0}^{t} e_{\theta}\right\|_{L^{\infty}\left(0, T ; H^{1}(\Omega)\right)}+\left\|e_{u}\right\|_{L^{\infty}\left(0, T ; H^{-1}(\Omega)\right)}=O\left(\tau^{\frac{1}{2}}\right) .
$$

We only deal with Dirichlet boundary conditions, though with some small modifications, our scheme could easily work for mixed type boundary conditions of the form (1.2) by following the technique presented in [15]. We only focus in the case where $p=2$, which is actually associated with the resistance welding model presented in Section 1, however our numerical scheme works efficiently for any exponent $p>0$.

\section{Stability of the numerical scheme}

In this section, we consider the stability of the time discretization scheme (3.10)- (3.12). To do this, we first introduce some assumptions for $f$ and $\beta$ in (1.1)- (1.3), see [16].

Assumption $\left(H_{\beta}\right): \beta: \mathbb{R} \rightarrow \mathbb{R}$ is nondecreasing and Lipschitz continuous function; more precisely

$$
0 \leq \beta^{\prime}(s) \leq L_{\beta}<+\infty, \quad \text { for a.e. } s \in \mathbb{R} .
$$

Moreover, $\beta$ satisfies $\beta(0)=0$ and grows at least linearlly at infinity, that is, there exist $c_{1}, c_{2}>0$ such that

$$
|s| \leq c_{1}+c_{2}|\beta(s)|, \quad \text { for any } \quad s \in \mathbb{R} .
$$

Assumption $\left(H_{f}\right): f: \mathbb{R} \rightarrow \mathbb{R}$ is a uniformly Lipschitz continuous function; that is,

$$
\left|f\left(s_{1}\right)-f\left(s_{2}\right)\right| \leq L_{f}\left|s_{1}-s_{2}\right|, \quad \forall s_{1}, s_{2} \in \mathbb{R} ;
$$

moreover

$$
f(s) \geq \sigma>0, \quad \forall s \in \mathbb{R} .
$$


Assumption $\left(H_{u_{0}}\right): u_{0} \in L^{\infty}(\Omega)$.

We shall choose the relaxation parameter $\mu$ in (3.10)- (3.12) such that $0<\mu \leq L_{\beta}^{-1}$, then the following property holds

$$
\alpha:=I-\mu \beta \quad \text { satisfies } \quad 0 \leq \alpha^{\prime}(s) \leq 1, \quad \text { for a.e. } s \in \mathbb{R} .
$$

Lemma 4.1. Let $U^{n}$ and $\Theta^{n}$ be the solutions of (3.10) - (3.12). Assume that $\left(H_{\beta}\right),\left(H_{f}\right)$ and $\left(H_{u_{0}}\right)$ hold. Then there exists a constant $C>0$ such that

$$
\max _{1 \leq n \leq N}\left\|\beta\left(U^{n}\right)\right\|_{L^{2}(\Omega)}+\sum_{n=1}^{N}\left\|U^{n}-U^{n-1}\right\|_{L^{2}(\Omega)}^{2}+\sum_{n=1}^{N} \tau\left\|\Theta^{n}\right\|_{H^{1}(\Omega)}^{2} \leq C .
$$

The proof of the Lemma 4.1 is given in the Appendix.

\section{Error estimates}

In this section, we will consider the error estimates of the time discretization scheme (3.10) - (3.12).

Theorem 5.1. Let $U^{n}$ and $\Theta^{n}$ be the solutions of (3.10) - (3.12). Let $u$ be the solution of (1.1) - (1.3). Assume that $\left(H_{\beta}\right),\left(H_{f}\right)$ and $\left(H_{u_{0}}\right)$ hold. Assume also that $u_{0} \in L^{\infty}(\Omega), \Delta \beta\left(u_{0}\right) \in L^{1}(\Omega)$ and in addition that

$$
\max _{1 \leq n \leq N}\left\|U^{n}\right\|_{L^{\infty}(\Omega)} \leq C .
$$

Then we have, with $\theta(t)=\beta(u(t)), e_{\theta}=\theta(t)-\Theta^{n}$ and $e_{u}=u(t)-U^{n}$ on $t \in\left(t_{n-1}, t_{n}\right)$,

$$
\left\|e_{\theta}\right\|_{L^{2}(\Omega \times(0, T))}+\left\|\int_{0}^{t} e_{\theta}\right\|_{L^{\infty}\left(0, T ; H^{1}(\Omega)\right)}+\left\|e_{u}\right\|_{L^{\infty}\left(0, T ; H^{-1}(\Omega)\right)}=O\left(\tau^{\frac{1}{2}}\right) .
$$

To prove Theorem 5.1, we need the following lemma (see (4.24) in [16]),

Lemma 5.2. If $u_{0} \in L^{\infty}(\Omega), \Delta \beta\left(u_{0}\right) \in L^{1}(\Omega)$, we have

$$
\sum_{i=1}^{N} \int_{t_{i-1}}^{t_{i}}\left(u(t), U^{i}-U^{i-1}\right) d t \leq C \tau
$$

Proof. The proof is similar to the proof of (4.24) in [16].

The proof of Theorem 5.1 is also given in Appendix.

\section{Numerical Experiments}

In this section, we present two numerical examples in order to test the efficiency of the numerical scheme inroduced in Section 3. For each example, we consider the approximate solutions both in local and nonlocal cases. We will use finite element method for spatial discretization and backward Euler method for time discretization.

Consider the following nonlocal problem, with $p>0$,

$$
\begin{aligned}
& \frac{\partial u}{\partial t}-\Delta \beta(u)=\frac{\lambda f(\beta(u))}{\left(\int_{\Omega} f(\beta(u)) d x\right)^{p}}, \quad \text { in } \Omega \times(0, T), \\
& -\frac{\partial \beta(u)}{\partial n}=k(x)(\beta(u)-q(x)), \quad \text { on } \partial \Omega \times(0, T), \\
& u(x, 0)=u_{0}(x), \quad \text { in } \Omega .
\end{aligned}
$$

For the functions involved in the mixed-type boundary condition (6.2) we take $k, q \in L^{2}(\partial \Omega)$ while $u_{0} \in L^{\infty}(\Omega)$. 
Denote $\theta=\beta(u)$. The variational form of (6.1)-(6.3) is to find $u(t) \in L^{2}(\Omega), \theta(t) \in H^{1}(\Omega)$, such that

$$
\begin{aligned}
\left(\frac{\partial u}{\partial t}, v\right)_{L^{2}(\Omega)} & +(\nabla \theta, \nabla v)_{L^{2}(\Omega)}+(k \theta, v)_{L^{2}(\partial \Omega)} \\
& =(k q, v)_{L^{2}(\partial \Omega)}+\frac{\lambda(f(\theta), v)_{L^{2}(\Omega)}}{\left(\int_{\Omega} f(\theta) d x\right)^{p}}, \quad \forall v \in H^{1}(\Omega) .
\end{aligned}
$$

For any $h>0$, let $\tau_{h}$ be a decomposition of $\Omega$ into triangles $\tau_{h}:\left\{T_{k}\right\}_{k=1}^{N_{h}}$ with diameters bounded by $h$, which stands for the mesh size. Let $\left\{\tau_{h}\right\}_{h}$ be a regular family of decompositions. We do not require either the quasi-uniformility or the acuteness of the family $\left\{\tau_{h}\right\}_{h}$. We have

$$
\Omega=\Omega_{h}=\bigcup_{k=1}^{K} T_{k} .
$$

Let

$$
\begin{aligned}
& M_{h}=\left\{\psi: \Omega_{h} \rightarrow \mathbb{R}:\left.\Psi\right|_{T_{k}} \text { is constant } \forall k=1,2, \ldots, K\right\}, \\
& S_{h}=\left\{\chi \in C^{0}\left(\Omega_{h}\right):\left.\chi\right|_{T_{k}} \text { is linear } \forall k=1,2, \ldots, K\right\} .
\end{aligned}
$$

Let $\Pi_{h}: C(\Omega) \rightarrow S_{h}$ denote the local linear interpolation operator. We define

$$
\langle\chi, \Phi\rangle_{h}=\sum_{k=1}^{K} \int_{T_{k}} \Pi_{h}(\chi \phi) d x .
$$

We also introduce the $L^{2}(\Omega)$-projection operator $P_{h}^{0}$ onto $M_{h}$ which, for any $v \in L^{2}(\Omega)$, is defined by

$$
\left(P_{h}^{0} v, \psi\right)=(v, \psi), \quad \forall \psi \in M_{h} .
$$

Let $\tau=T / N$ be the time step ( $N$ is a positive integer) and $t_{n}=n \tau$. We define the following finite element method for solving (6.1)-(6.3). Find $U^{n} \in M_{h}, \Theta^{n} \in S_{h}$, where $U^{n} \approx u\left(t_{n}\right), \Theta^{n} \approx \beta\left(u\left(t_{n}\right)\right)=\theta\left(t_{n}\right)$, such that, for any $\chi \in S_{h}$,

$$
\begin{aligned}
& U^{0}=P_{h}^{0} u_{0}, \\
& \begin{array}{l}
\left\langle\Theta^{n}, \chi\right\rangle_{h}+\frac{\tau}{\mu}\left(\nabla \Theta^{n}, \nabla \chi\right)_{L^{2}(\Omega)}+\frac{\tau}{\mu}\left(k \Theta^{n}, \chi\right)_{L^{2}(\partial \Omega)} \\
\quad=\frac{\tau}{\mu}(k q, \chi)_{L^{2}(\partial \Omega)}+\left(\beta\left(U^{n-1}\right), \chi\right)_{L^{2}(\Omega)}+\frac{\tau}{\mu} \frac{\lambda\left(f\left(\beta\left(U^{n-1}\right)\right), \chi\right)_{L^{2}(\Omega)}}{\left(\int_{\Omega} f\left(\beta\left(U^{n-1}\right)\right) d x\right)^{p}},
\end{array} \\
& U^{n}=U^{n-1}+\mu\left[P_{h}^{0} \Theta^{n}-\beta\left(U^{n-1}\right)\right],
\end{aligned}
$$

where $\mu: 0<\mu \leq L_{\beta}^{-1}$ is a fixed number (the so-called relaxation parameter).

Let $\varphi_{j}, j=1,2, \ldots N_{h}$ be the basis functions on the nodes $P_{j}, j=1,2, \ldots, N_{h}$ on $S_{h}$. Assume that

$$
\Theta^{n}=\sum_{j=1}^{N_{h}} \theta_{j}^{n} \varphi_{j}
$$

and choose $\chi=\varphi_{l}, l=1,2 \ldots, N_{h}$ in (7.5) then we get

$$
\begin{gathered}
\left\langle\sum_{j=1}^{N_{h}} \theta_{j}^{n} \varphi_{j}, \varphi_{l}\right\rangle_{h}+\frac{\tau}{\mu}\left(\nabla \sum_{j=1}^{N_{h}} \theta_{j}^{n} \varphi_{j}, \nabla \varphi_{l}\right)_{L^{2}(\Omega)}+\frac{\tau}{\mu}\left(k \sum_{j=1}^{N_{h}} \theta_{j}^{n} \varphi_{j}, \varphi_{l}\right)_{L^{2}(\partial \Omega)} \\
=\frac{\tau}{\mu}\left(k g, \varphi_{l}\right)_{L^{2}(\partial \Omega)}+\left(\beta\left(U^{n-1}\right), \varphi_{l}\right)_{L^{2}(\Omega)}+\frac{\tau}{\mu} \frac{\lambda\left(f\left(\beta\left(U^{n-1}\right)\right), \varphi_{l}\right)_{L^{2}(\Omega)}}{\left(\int_{\Omega} f\left(\beta\left(U^{n-1}\right)\right) d x\right)^{p}}
\end{gathered}
$$


Denote

$$
\begin{gathered}
\mathbf{M}=\left(\varphi_{j}, \varphi_{l}\right)_{L^{2}(\Omega)} \quad \mathbf{K}=\left(k \varphi_{j}, \varphi_{l}\right)_{L^{2}(\partial \Omega)}, \quad \mathbf{G}=\left(k q, \varphi_{l}\right)_{L^{2}(\partial \Omega)}, \\
\mathbf{B}=\left(\beta\left(U^{n-1}\right), \varphi_{l}\right)_{L^{2}(\Omega)}, \quad \mathbf{F}=\left(f\left(\Theta^{n-1}\right), \varphi_{l}\right)_{L^{2}(\Omega)}, \quad \Theta^{n}=\left(\theta_{j}^{n}\right),
\end{gathered}
$$

and $Q=\left(\int_{\Omega} f\left(\Theta^{n-1}\right) d x\right)^{p}$. We have the matrix form

$$
\left(\mathbf{M}+\frac{\tau}{\mu} \mathbf{S}+\frac{\tau}{\mu} \mathbf{K}\right) \Theta^{n}=\frac{\tau}{\mu} \mathbf{G}+\mathbf{B}+\frac{\tau}{\mu} \frac{\lambda \mathbf{F}}{Q} .
$$

When we obtain $\Theta^{n}$, we can calculate $U^{n}$ from (6.7).

We use the following steps to calculate $\mathbf{B}, \mathbf{F}$ and $Q$.

For the calculation of $\mathbf{B}$, we note that

$$
\left(\beta\left(U^{n-1}\right), \varphi_{l}\right)_{L_{2}(\Omega)}=\int_{\Omega} \beta\left(U^{n-1}\right) \varphi_{l} d x=\sum_{k=1}^{N_{h}} \int_{T_{k}} \beta\left(U^{n-1}\right) \varphi_{l} d x,
$$

where $\beta\left(U^{n-1}\right)$ is a piecewise constant function and

$$
\int_{T_{k}} \varphi_{l} d x=\left\{\begin{array}{l}
\left|T_{k}\right| / 3, \quad \text { if } P_{l} \text { is a node of } T_{k}, \\
0, \quad \text { otherwise }
\end{array}\right.
$$

for $\left|T_{k}\right|$ denoting the area of $T_{k}$.

To calculate $\mathbf{F}$, we note that

$$
\begin{aligned}
\left(f\left(\Theta^{n-1}\right), \varphi_{l}\right)_{L^{2}(\Omega)} & =\left(f\left(\sum_{j=1}^{N_{h}} \theta_{j}^{n-1} \varphi_{j}\right), \varphi_{l}\right)_{L^{2}(\Omega)} \\
& \approx \sum_{j=1}^{N_{h}} f\left(\theta_{j}^{n-1}\right)\left(\varphi_{j}, \varphi_{l}\right)_{L^{2}(\Omega)}=\left(\mathbf{M} * f\left(\Theta^{n-1}\right)\right)_{l} .
\end{aligned}
$$

Finally, we have

$$
\begin{aligned}
Q & =\left(\int_{\Omega} f\left(\Theta^{n-1}\right) d x\right)^{p}=\left(\int_{\Omega} f\left(\sum_{j=1}^{N_{h}} \theta_{j}^{n-1} \varphi_{j}\right) d x\right)^{p} \\
& =\left(\sum_{j=1}^{N_{h}} f\left(\theta_{j}^{n-1}\right) \cdot \int_{\Omega} \varphi_{j} d x\right)^{p}
\end{aligned}
$$

where $\int_{\Omega} \varphi_{j} d x=\sum_{k=1}^{N_{h}} \int_{T_{k}} \varphi_{j} d x$.

Therefore we have built the following algorithm $(\mathcal{A})$ :

Step 1: Find $U^{0}=P_{h}^{0} u_{0}, u_{0}$ is the initial value.

Step 2: Find $\beta\left(U^{0}\right)$.

Step 3: Find $\Theta^{1}$ by (7.5).

Step 4: Find $U^{1}$ by (6.7).

Step 5: Go to Steps 1-4 to find next $\Theta^{n}, U^{n}, n=2,3, \ldots$.

In the following we present two examples focusing on the two-dimensional case, which according to the modelling in Section 2, is very interesting from the application point of view. 


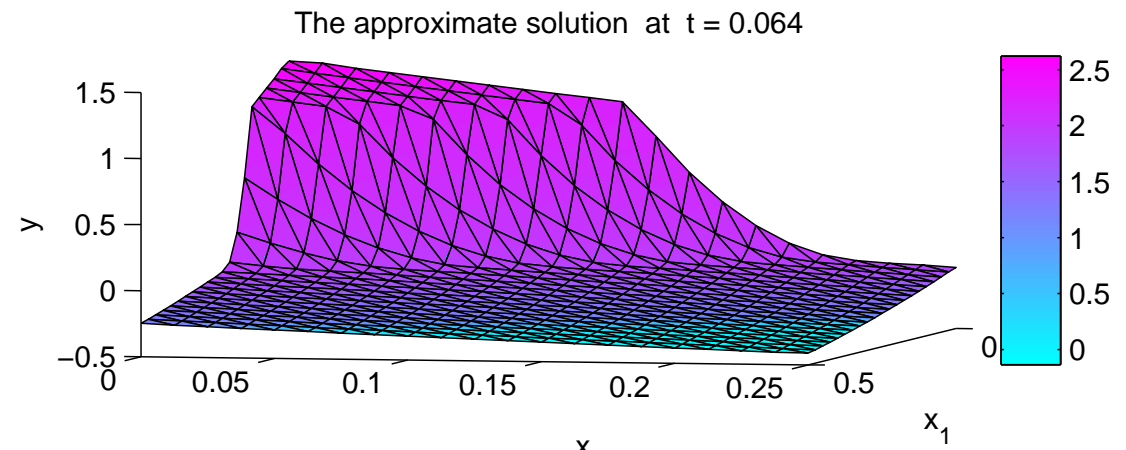

The approximate solutien at $t=0.129$

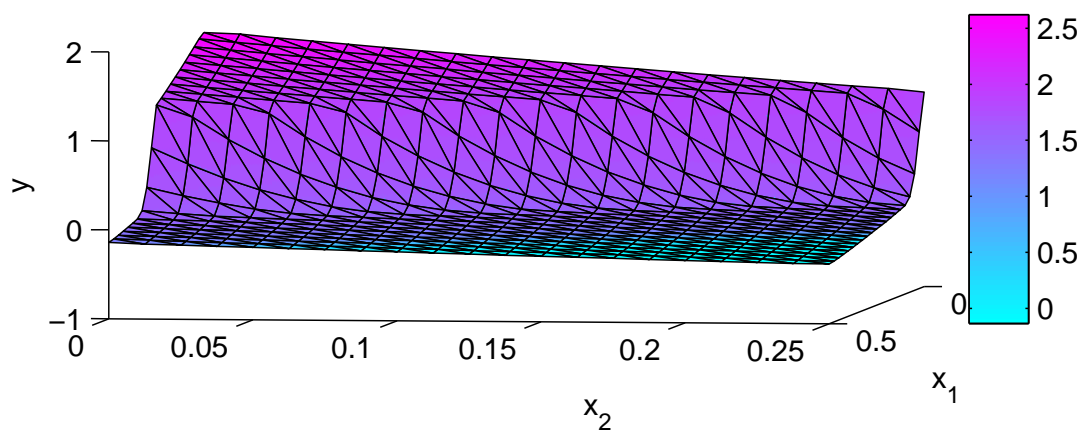

FiguRE 3. Formation of interface for Example 1 when $f(s)=0$ at different times.
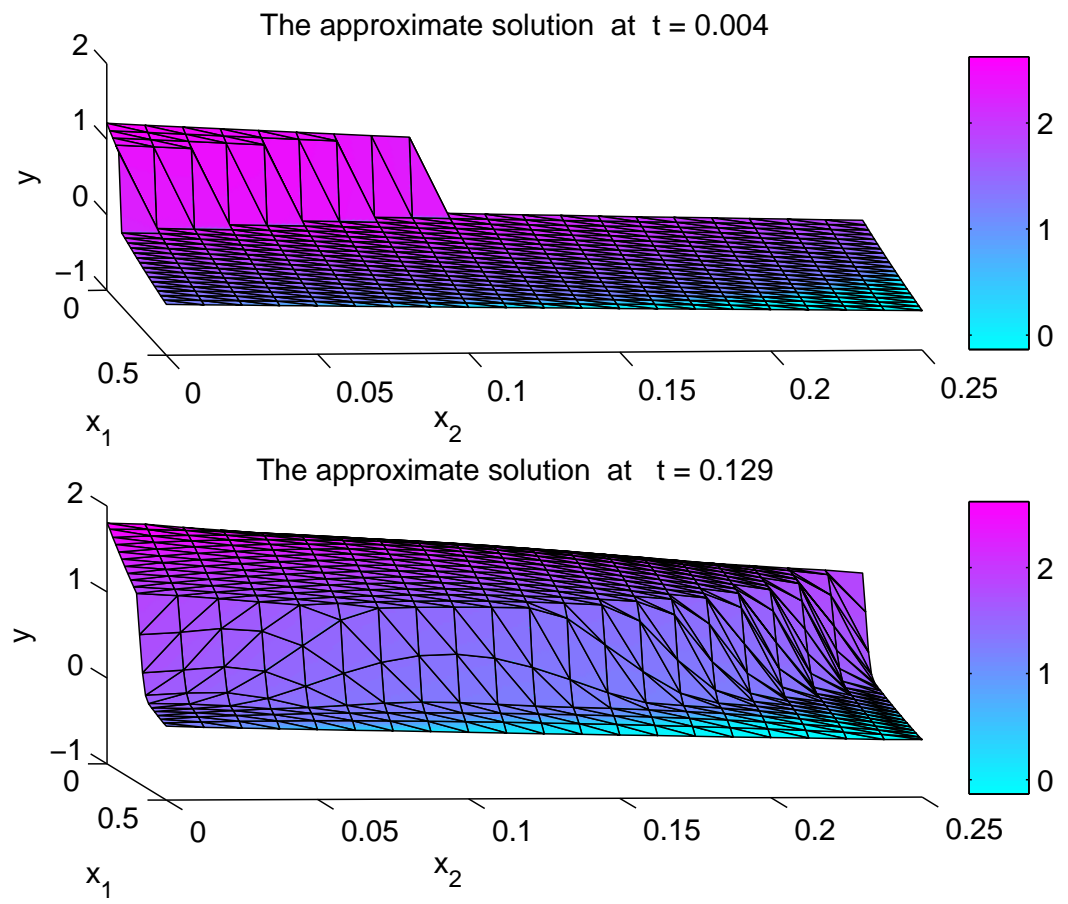

Figure 4 . Formation of interface for Example 1 when $f(s)=s^{2}+1$ and $\lambda=1, p=2$ at different times. 
Example 6.1. [4], [16], [15], [23]

Let $\Omega=(0,0.5) \times(0,0.25), 0<t<T=0.25$ and

$$
\beta(u)=\left\{\begin{array}{l}
u, \quad \text { if } \quad u<0 \\
0, \quad \text { if } \quad 0 \leq u \leq 1 \\
u-1, \quad \text { if } u>1
\end{array}\right.
$$

When $f=0$, the exact solution of (6.1)-(6.3) is

$$
u(x, y, t)=\left\{\begin{array}{l}
2\left[e^{\Phi(x, y, t)}-1\right]+1, \quad \text { if } \quad \Phi(x, y, t) \geq 0 \\
e^{\Phi(x, y, t)}-1, \quad \text { if } \quad \Phi(x, y, t)<0
\end{array}\right.
$$

where

$$
\Phi(x, y, t)=-x-y+2 t+0.1=0,
$$

is the interface (free boundary). Dirichlet data are prescribed on the boundary $\Gamma$.

Let $M_{1}$ and $M_{2}$ be any positive integrers. Let $0=x_{0}<x_{1}<x_{2}<\cdots<x_{M_{1}}=0.5$ be the partition of the interval $[0,0.5]$ and $h_{x}=0.5 / M_{1}$ the stepsize. Similarly $0=y_{0}<y_{1}<y_{2}<\cdots<y_{M_{2}}=0.25$ is a partition of the interval $[0,0.25]$ and $h_{y}=0.25 / M_{2}$ the stepsize. We divide the rectangle $\Omega=$ $[0,0.5] \times[0,0.25]$ into the triangles $T_{k}, k=1,2, \ldots, N_{h}$ with the same size. The length of the longest side of each triangle is then $h=\sqrt{h_{x}^{2}+h_{y}^{2}}$. Let now $0=t_{0}<t_{1}<t_{2}<\cdots<t_{N}=T$ be the time partition of $[0, T]$ and $\tau$ the time stepsize. We will choose $\tau=C^{*} h$ for any constant $C^{*}$ in our numerical simulations below.

The authors in [16] proved the error estimates both for time and space discretizations for local problem (3.1) -(3.3) and their numerical experiments show that the numerical results are consistent with the theoretical results. In the current paper we only prove the error estimates for the time discretization for the non-local problem (6.1)-(6.3). However we believe that one can obtain similar error estimates for the space discretization as for the local problem in [16]. We will evaluate the actual order of convergence of both variables $u$ and $\theta$ in our numerical experiments.

Denote

$$
E_{\theta}^{h}:=\left\{\tau \sum_{n=1}^{N} \sum_{k=1}^{N_{h}} \int_{T_{k}} \Pi_{h}\left(\left[\Theta^{n}-\theta^{n}\right]^{2}\right) d x\right\}^{\frac{1}{2}},
$$

and analogously for $E_{u}^{h}$. Assume that $E_{u}^{h}=C h^{p_{u}}$ and $E_{\theta}^{h}=C h^{p_{\theta}}$, then we have

$$
p_{u}=\frac{\log \left(E_{u}^{h_{1}} / E_{u}^{h_{2}}\right)}{\log \left(h_{1} / h_{2}\right)}, \quad p_{\theta}=\frac{\log \left(E_{\theta}^{h_{1}} / E_{\theta}^{h_{2}}\right)}{\log \left(h_{1} / h_{2}\right)} .
$$

Since we do not know the exact solution $\theta^{n}$ and $u^{n}$ for our non-local problem, in order to check the estimated convergence orders $p_{u}$ and $p_{\theta}$, we assume that the exact solutions $\theta^{n}$ and $u^{n}$ are obtained by using a very small mesh size $h$ with $M_{1}=M_{2}=40$ which implies that the number of elements is $2 \times M_{1} \times M_{2}=3200$. We then choose $M_{1}=M_{2}=5,10,15,20$ respectively and calculate the approximate solutions $\Theta^{n}$ and $U^{n}$. The estimated convergence orders $p_{\theta}$ and $p_{u}$ are listed in Table 1 . We note that $p_{\theta} \approx \frac{1}{2}$ and $p_{u} \approx \frac{1}{4}$ which are consistent with the error estimates for the local problem in [16].

According to the general theory the formation of a moving boundary is expected for problem (6.1)(6.3). As mentioned in Section 2 it actually describes the mushy region formulated between the two phases (liquid and solid) in the contact area during the welding process. In Figure 3, the discrete interfaces at different times with $f=0$ for Example 1 are shown where we choose $\tau=0.25 / 70$ and $M_{1}=M_{2}=20$. Figure 4 depicts the formation of the interface at different times for $f(s)=s^{2}+1$ when $\lambda=1, p=2$ 
The approximate solution at $\mathrm{t}=0.097$

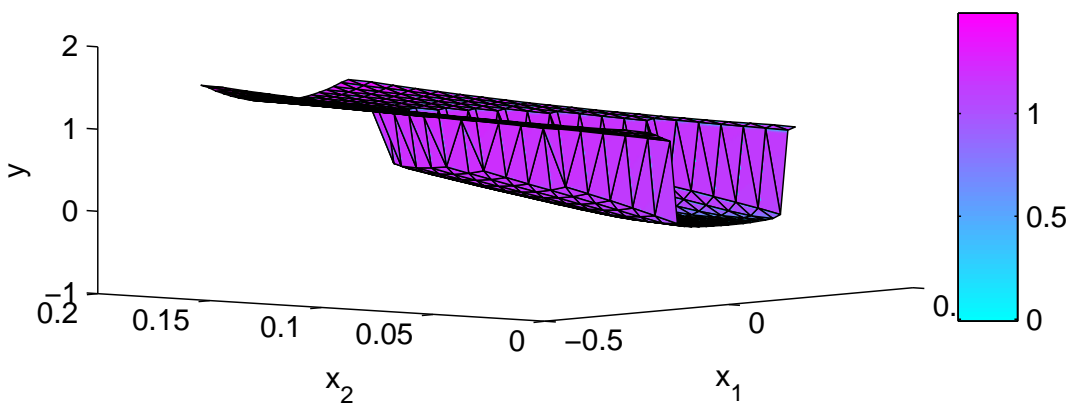

The approximate solution at $t=0.200$

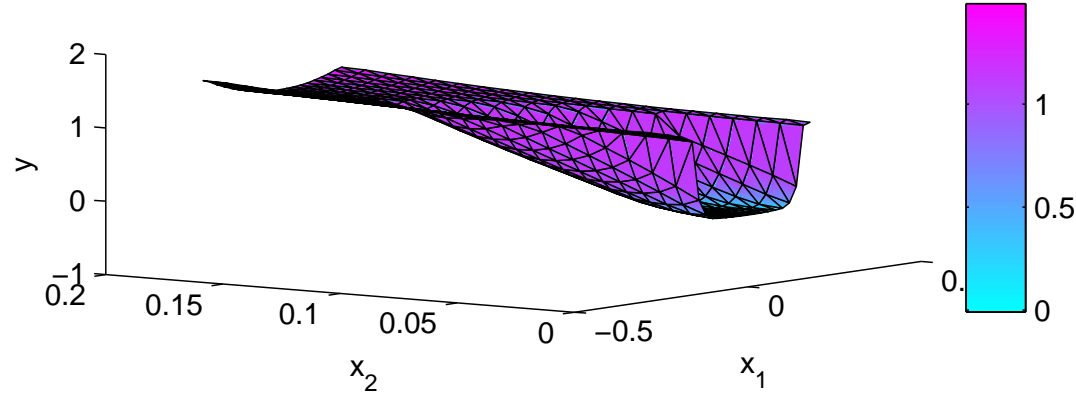

Figure 5. Formation of interface for Example 2 when $f(s)=0$ at different times.

The approximate solution at $\mathrm{t}=0.006$

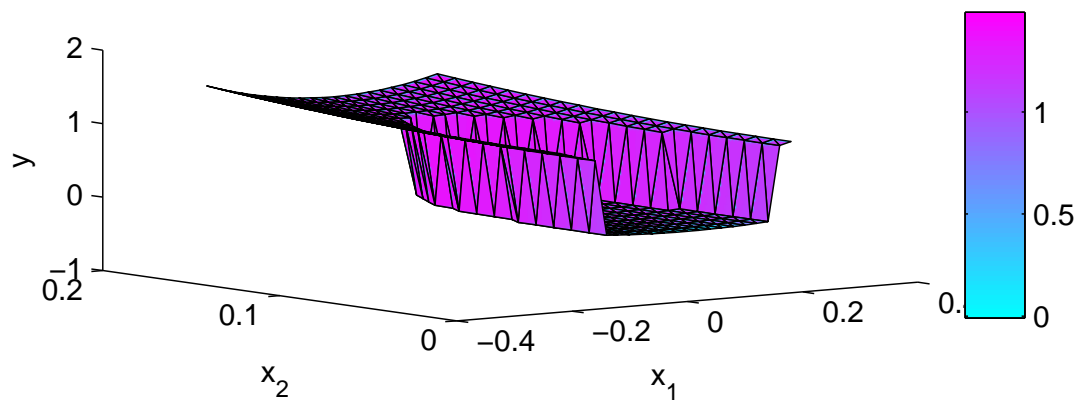

The approximate solution at $t=0.097$

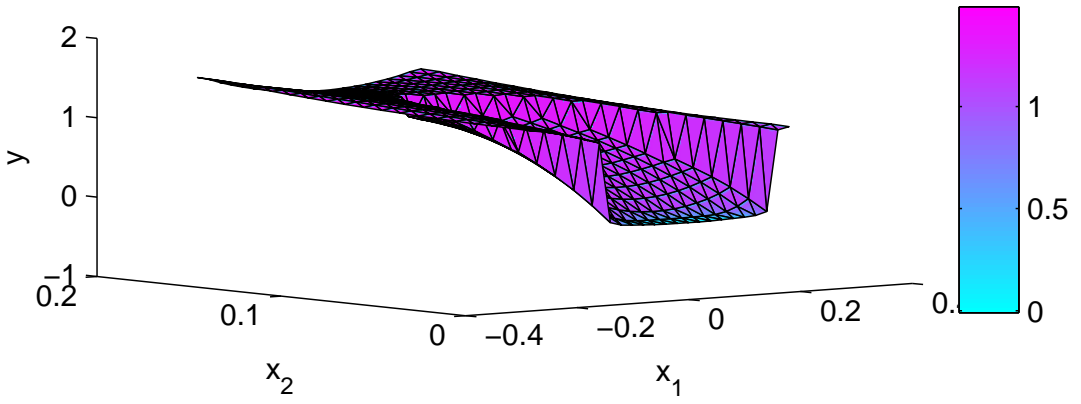

FiguRE 6 . Formation of interface for Example 2 when $f(s)=f_{e}(s)$ and $\lambda=0.2, p=2$ at different times. 


\begin{tabular}{|c|c|c|c|c|c|}
\hline$M_{1}\left(=M_{2}\right)$ & Nel & $E_{u}$ & $E_{\theta}$ & $p_{u}$ & $p_{\theta}$ \\
\hline 5 & 50 & 0.062 & 0.00635 & & \\
\hline 10 & 200 & 0.0547 & 0.0042 & 0.18 & 0.60 \\
\hline 15 & 450 & 0.0482 & 0.0033 & 0.31 & 0.57 \\
\hline 20 & 800 & 0.0447 & 0.0028 & 0.26 & 0.55 \\
\hline
\end{tabular}

TABLE 1. The estimated order of convergence for Example 1 at $T=0.25$.

for $\tau=0.25 / 70$ and $M_{1}=M_{2}=20$. It is worth mentioning that due to the external disturbance $f \neq 0$ the interface moves faster compared to the case $f=0$. Furthermore, as $\lambda$, or equivalently the applied voltage, increases then again the evolution of the moving boundary in time becomes faster.

Example 6.2. [16], [15], [23]

Let $\Omega=(-0.22,0.18) \times(0,0.2), 0<t<T=0.4$ and

$$
\beta(u)=\left\{\begin{array}{l}
\frac{1}{2} u, \quad \text { if } \quad u<0, \\
0, \quad \text { if } \quad 0 \leq u \leq 1, \\
\frac{1}{3}(u-1), \quad \text { if } u>1 .
\end{array}\right.
$$

When $f=0$, the exact solution of $(6.1)$ - (6.3) is

$$
u(x, y, t)=\left\{\begin{array}{l}
6 \Phi(x, y, t)+1, \quad \text { if } \Phi(x, y, t) \geq 0 \\
2 \Phi(x, y, t), \quad \text { if } \Phi(x, y, t)<0,
\end{array}\right.
$$

where

$$
\Phi(x, y, t)=\left(x^{2}+y^{2}-e^{-4 t}\right) / 4 e^{2.4}=0,
$$

is the free boundary. Dirichlet data are prescribed on the boundaries.

We use the same notations as in Example 1. In Figure 5, the discrete interfaces at different times with $f=0$ for Example 2 are shown where we choose $\tau=0.25 / 70$ and $M_{1}=M_{2}=20$.

Experimental data show that the electrical conductivity, denoted by $f(s)$, usually has a discontinuous profile, see also Section 2.But a discontinuous function does not satisfy Assumption $\left(H_{f}\right)$ and hence the developed algorithm in Section 3 is not applicable in this case. However, algorithm $(\mathcal{A})$ can still provide reliable numerical experiments in this delicate case. Therefore in the current example, in order to be consistent with applications, we consider the following discontinuous function

$$
f_{e}(s)= \begin{cases}e^{s^{2}}+1, & \text { if } s \geq 0 \\ e^{s^{2}}+2, & \text { if } s<0 .\end{cases}
$$

Following the same approach to estimate $p_{u}$ and $p_{\theta}$ as in Example 1 we finally derive Table 2. We also note that $p_{\theta} \approx \frac{1}{2}$ and $p_{u} \approx \frac{1}{4}$ which are consistent with the error estimates obtained for the local problems. [16].

Also Figure 6 depicts the formation of this interface at different times for $f(s)=s^{2}+1$ when $\lambda=1, p=2$ for $\tau=0.25 / 70$ and $M_{1}=M_{2}=20$. It is worth mentioning that due to the external disturbance $f \neq 0$ the interface moves faster compared to the case $f=0$. Furthermore, as $\lambda$, or equivalently the applied voltage, increases then again the moving boundary moves faster.

Acknowledgements. We would like to thank the anonymous referees for their stimulating comments which actually improved the presentation of the current work. 


\begin{tabular}{|c|c|c|c|c|c|}
\hline$M_{1}\left(=M_{2}\right)$ & $\mathrm{Nel}$ & $E_{u}$ & $E_{\theta}$ & $p_{u}$ & $p_{\theta}$ \\
\hline 5 & 50 & 0.0622 & 0.00135 & & \\
\hline 10 & 200 & 0.0516 & 0.0010 & 0.27 & 0.43 \\
\hline 15 & 450 & 0.0478 & $8.1319 \mathrm{e}-004$ & 0.19 & 0.51 \\
\hline 20 & 800 & 0.0441 & $7.1035 \mathrm{e}-004$ & 0.28 & 0.47 \\
\hline
\end{tabular}

TABLE 2. The estimated order of convergence in Example 2 at $T=0.4$.

\section{References}

[1] M. R. Sidi Ammi, O. Mul. Error estimates for the Chernoff scheme to approximate a nonlocal problem, Proc. Estonian Acad. Sci. Phys. Maths., 56 (2007), 359-372.

[2] S. N. Antontsev, M. Chipot. The analysis of blow-up for the thermistor problem, Siberian Math. J., 38 (1997), 827-841.

[3] J. Bebernes, P. Talaga. Nonlocal problems modelling shear banding, Comm. Appl. Nonlinear Analysis, 3, (1996), 79-103.

[4] A. E. Berger, H. Brezis, J. C. W. Rogers. A numerical method for solving the problem $u_{t}-\Delta f(u)=0$, RAIRO Anal. Numer., 13, (1979), 297-312.

[5] M. Brokate, J. Sprekels. Hysteresis and Phase Transitions Appl.Math. Sci. Springer: New York, 1996.

[6] I.J. Hewitt, A.A. Lacey, R.I. Todd. A Mathematical Model for flash sintering, to appear in Math. Model. Nat. Phenom.

[7] D. Hömberg, E. Rocca. A model for resistance welding including phase transitions and Joule heating, Math. Meth. Appl. Sciences, 34 (2011), 2077-2088.

[8] N. I. Kavallaris. Asymptotic behaviour and blow-up for a nonlinear diffusion problem with a non-local source term, Proc. Edinb. Math. Soc., 47 (2004), 375-395.

[9] N. I. Kavallaris, A.A. Lacey, C.V. Nikolopoulos, C. Voong. Behaviour of a non-local equation modelling linear friction welding, IMA J. Appl. Mathematics, 72 (2007), 597-616.

[10] N. I. Kavallaris, T. Nadzieja. On the blow-up of the non-local thermistor problem, Proc. Edinb. Math. Soc., 50 (2007), 389-409.

[11] N.I. Kavallaris, T. Suzuki. On the finite-time blow-up of a non-local parabolic equation describing chemotaxis, Differential Integral Equations, 20, (2007), 293-308.

[12] A. A. Lacey. Thermal runaway in a nonlocal problem modelling ohmic heating, Part I, Model derivation and some special cases, European. J. Appl. Math., 6 (1995), 127-144.

[13] E.A. Latos, D. E. Tzanetis. Existence and blow-up of solutions for a non-local filtration and porous medium problem, Proc. Edinb. Math. Soc., 53 (2010), 195-209.

[14] E.A. Latos, D. E. Tzanetis. Grow-up of critical solutions for a non-local porous medium problem with Ohmic heating source, Nonlinear Differ. Equ. Appl., 17 (2010), 137-151.

[15] R. H. Nochetto, C. Verdi. Approximation of degenerate parabolic problems using numerical integration, SIAM J. Numer. Anal., 25, (1988), 784-814.

[16] R. H. Nochetto, C. Verdi. An efficient linear scheme to approximate parabolic free boundary problems: Error estimates and implementation, Mathematics of Computation, 51 (1988), 27-53.

[17] N. Saunders, X. Li, A. P. Miodownik, J-Ph. Schillé. Modelling of the thermo-physical and physical properties relevant to solidification, In Advanced Solidification Processes X, Stefanescu D, Warren JA, Jolly MR, Krane MJM (eds). TMS: Warrendale, PA, (2003); 669.

[18] I. Steinbach, M. Apel. Multi phase field model for solid state transformation with elastic strain, Physica D, 217 (2006), $153-160$

[19] D. E. Tzanetis. Blow-up of radially symmetric solutions of a non-local problem modelling ohmic heating, Electron. J. Diff. Eqns, 11 (2002), 1-26.

[20] D. E. Tzanetis, P. M. Vlamos. A nonlocal problem modelling ohmic heating with variable thermal conductivity, Nonlin. Analysis, 2 (2001), 443-454.

[21] D. E. Tzanetis, P. M. Vlamos. Some interesting special cases of a non-local problem modelling Ohmic heating with variaable thermal conductivity, Proc. Edinb. Math. Soc., 44 (2001), 58559-5.

[22] J.L. Vazquez. The Porous Medium Equation: Mathematical Theory, Oxford Science Publications, 2007.

[23] C. Verdi. On the numerical approach to a two-phase Stefan problem with non-linear flux, Calcolo, 22 (1985), 351-381.

[24] G. Wolansky. A critical parabolic estimate and application to non-local equations arising in chemotaxis, Appl. Anal., 66 (1997), 291-321.

[25] Ya. B. Zel'dovich, Yu.P. Raizer. Physics of Shock Wavesand High-Temperature Hydrodynamic Phenomena, Vol. II, Academic Press, New York. 


\section{Appendix}

In this section we give the proofs of Lemma 4.1 and Theorem 5.1.

Proof of Lemma 4.1. Denote $F\left(\beta\left(U^{n-1}\right)\right)=\frac{\left(f\left(\beta\left(U^{n-1}\right)\right), v\right)}{\left(\int_{\Omega} f\left(\beta\left(U^{n-1}\right)\right) d x\right)^{2}}$. The equations (3.10) - (3.12) can be rewritten as, with $\partial U^{n}=\left(U^{n}-U^{n-1}\right) / \tau$,

$$
\left(\partial U^{n}, v\right)+\left(\nabla \Theta^{n}, \nabla v\right)=\left(F\left(\beta\left(U^{n-1}\right)\right), v\right), \quad \text { for any } v \in H_{0}^{1}(\Omega) .
$$

Choose $v=\tau \Theta^{n}$ in (7.1) and sum up from 1 to $N$, we get

$$
\sum_{n=1}^{N}\left(\partial U^{n}, \tau \Theta^{n}\right)+\sum_{n=1}^{N}\left(\nabla \Theta^{n}, \tau \Theta^{n}\right)=\sum_{n=1}^{N}\left(F\left(\beta\left(U^{n-1}\right)\right), \tau \Theta^{n}\right) .
$$

Note that, by (4.2),

$$
\begin{aligned}
\Theta^{n} & =\frac{1}{\mu}\left(U^{n}-U^{n-1}\right)+\beta\left(U^{n-1}\right)=\frac{1}{\mu}\left(U^{n}-U^{n-1}\right)+\frac{1}{\mu}\left(U^{n-1}-\alpha\left(U^{n-1}\right)\right) \\
& =\frac{1}{\mu} U^{n}-\frac{1}{\mu} \alpha\left(U^{n-1}\right) \\
& =\frac{1}{2} \beta\left(U^{n}\right)+\frac{1}{2 \mu} U^{n}+\frac{1}{2 \mu}\left(\alpha\left(U^{n}\right)-\alpha\left(U^{n-1}\right)\right)-\frac{1}{2 \mu} \alpha\left(U^{n-1}\right),
\end{aligned}
$$

and thus we have

$$
\begin{aligned}
& 2 \sum_{n=1}^{N}\left(\partial U^{n}, \tau \Theta^{n}\right)=2 \sum_{n=1}^{N}\left(U^{n}-U^{n-1}, \Theta^{n}\right) \\
& =2 \sum_{n=1}^{N}\left(U^{n}-U^{n-1}, \frac{1}{2} \beta\left(U^{n}\right)\right)+2 \sum_{n=1}^{N}\left(U^{n}-U^{n-1},-\frac{1}{2 \mu} \alpha\left(U^{n-1}\right)\right) \\
& +2 \sum_{n=1}^{N}\left(U^{n}-U^{n-1}, \frac{1}{2 \mu} U^{n}\right)+2 \sum_{n=1}^{N}\left(U^{n}-U^{n-1}, \frac{1}{2 \mu}\left(\alpha\left(U^{n}\right)-\alpha\left(U^{n-1}\right)\right)\right) .
\end{aligned}
$$

Given a function $\pi: \mathbb{R} \rightarrow \mathbb{R}, \Phi_{\pi}$ stands for the convex function defined by

$$
\Phi_{\pi}(s)=\int_{0}^{s} \pi(z) d z, \quad \text { for } s \in \mathbb{R} .
$$

We have, since $\beta^{\prime}(s)>0$ and $\alpha^{\prime}(s)>0$, then

$$
\begin{gathered}
\Phi_{\beta}\left(U^{n}\right)-\Phi_{\beta}\left(U^{n-1}\right)=\Phi_{\beta}^{\prime}(c)\left(U^{n}-U^{n-1}\right) \\
=\beta(c)\left(U^{n}-U^{n-1}\right) \leq \beta\left(U^{n}\right)\left(U^{n}-U^{n-1}\right), \\
\Phi_{\alpha}\left(U^{n}\right)-\Phi_{\alpha}\left(U^{n-1}\right)=\Phi_{\alpha}^{\prime}(c)\left(U^{n}-U^{n-1}\right) \\
=\alpha(c)\left(U^{n}-U^{n-1}\right) \geq \alpha\left(U^{n-1}\right)\left(U^{n}-U^{n-1}\right), \\
\left(U^{n}-U^{n-1}, \alpha\left(U^{n}\right)-\alpha\left(U^{n-1}\right)\right) \geq 0 .
\end{gathered}
$$


Further, using the equality $2 a(a-b)=a^{2}-b^{2}+(a-b)^{2}$,

$$
\begin{aligned}
& 2 \sum_{n=1}^{N}\left(\partial U^{n}, \tau \Theta^{n}\right) \geq \int_{\Omega} \sum_{n=1}^{N}\left\{\left[\Phi_{\beta}\left(U^{n}\right)-\Phi_{\beta}\left(U^{n-1}\right)\right]\right. \\
& \left.+\frac{1}{\mu}\left[\Phi_{\alpha}\left(U^{n-1}\right)-\Phi_{\alpha}\left(U^{n}\right)\right]\right\} d x+\frac{1}{\mu} \sum_{n=1}^{N}\left(U^{n}-U^{n-1}, U^{n}\right) \\
& =\int_{\Omega} \sum_{n=1}^{N}\left\{\left[\Phi_{\beta}\left(U^{n}\right)-\Phi_{\beta}\left(U^{n-1}\right)\right]+\frac{1}{\mu}\left[\Phi_{\alpha}\left(U^{n-1}\right)-\Phi_{\alpha}\left(U^{n}\right)\right]\right\} d x \\
& +\frac{1}{\mu}\left[\left\|U^{n}\right\|_{L^{2}(\Omega)}^{2}-\left\|U^{0}\right\|_{L^{2}(\Omega)}^{2}+\sum_{n=1}^{N}\left\|U^{n}-U^{n-1}\right\|_{L^{2}(\Omega)}^{2}\right] .
\end{aligned}
$$

Since $\frac{1}{2 L_{\beta}} \beta^{2}(s) \leq \Phi_{\beta}(s) \leq \frac{L_{\beta}}{2} s^{2}$ and $0 \leq \beta^{\prime}(s) \leq L_{\beta}$, we have

$$
\begin{aligned}
\int_{\Omega} \sum_{n=1}^{N}\left[\Phi_{\beta}\left(U^{n}\right)-\Phi_{\beta}\left(U^{n-1}\right)\right] d x & =\int_{\Omega}\left[\Phi_{\beta}\left(U^{N}\right)-\Phi_{\beta}\left(U^{0}\right)\right] \\
& \geq \frac{1}{2 L_{\beta}}\left\|\beta\left(U^{N}\right)\right\|_{L^{2}(\Omega)}^{2}-\frac{L_{\beta}}{2}\left\|U^{0}\right\|_{L^{2}(\Omega)}^{2}
\end{aligned}
$$

Also since $\frac{1}{2 L_{\alpha}} \alpha^{2}(s) \leq \Phi_{\beta}(s) \leq \frac{L_{\alpha}}{2} s^{2}$ and $0 \leq \beta^{\prime}(s) \leq 1$, we obtain

$$
\begin{aligned}
\frac{1}{\mu} \int_{\Omega} \sum_{n=1}^{N}\left[\Phi_{\alpha}\left(U^{n-1}\right)-\Phi_{\alpha}\left(U^{n}\right)\right] d x & =\frac{1}{\mu} \int_{\Omega}\left[\Phi_{\alpha}\left(U^{0}\right)-\Phi_{\alpha}\left(U^{N}\right)\right] \\
& \geq \frac{1}{2 \mu}\left\|\alpha\left(U^{0}\right)\right\|_{L^{2}(\Omega)}^{2}-\frac{1}{2 \mu}\left\|U^{N}\right\|_{L^{2}(\Omega)}^{2}
\end{aligned}
$$

Therefore

$$
\begin{aligned}
& 2 \sum_{n=1}^{N}\left(U^{n}-U^{n-1}, \Theta^{n}\right) \\
& \geq \frac{1}{2 L_{\beta}}\left\|\beta\left(U^{N}\right)\right\|_{L^{2}(\Omega)}^{2}-\frac{L_{\beta}}{2}\left\|U^{0}\right\|_{L^{2}(\Omega)}^{2}+\frac{1}{2 \mu}\left\|\alpha\left(U^{0}\right)\right\|_{L^{2}(\Omega)}^{2}-\frac{1}{2 \mu}\left\|U^{N}\right\|_{L^{2}(\Omega)}^{2} \\
& +\frac{1}{2 \mu}\left[\left\|U^{N}\right\|_{L^{2}(\Omega)}^{2}-\left\|U^{0}\right\|_{L^{2}(\Omega)}^{2}+\sum_{n=1}^{N}\left\|U^{n}-U^{n-1}\right\|_{L^{2}(\Omega)}^{2}\right] \\
& \geq-C+C\left\|\beta\left(U^{N}\right)\right\|_{L^{2}(\Omega)}^{2}+\frac{1}{2 \mu}\left\|U^{n}-U^{n-1}\right\|_{L^{2}(\Omega)}^{2} .
\end{aligned}
$$

We next consider the nonlocal term $\sum_{n=1}^{N}\left(F\left(\beta\left(U^{n-1}\right)\right), \tau \Theta^{n}\right)$. Since $f$ satisfies Lipschitz condition $\left(H_{f}\right)$ and $f(s) \geq \sigma>0$ for $s \in \mathbb{R}$, we have

$$
\left|F\left(\beta\left(U^{n-1}\right)\right)\right|=\left|\frac{\lambda f\left(\beta\left(U^{n-1}\right)\right)}{\left(\int_{\Omega} f\left(\beta\left(U^{n-1}\right)\right) d x\right)^{2}}\right| \leq C\left|f\left(\beta\left(U^{n-1}\right)\right)\right| \leq C\left(1+\left|\beta\left(U^{n-1}\right)\right|\right) .
$$


Hence, noting that $\Theta^{n}=\frac{1}{\mu}\left(U^{n}-U^{n-1}\right)+\beta\left(U^{n-1}\right)$,

$$
\begin{aligned}
& \sum_{n=1}^{N}\left(F\left(\beta\left(U^{n-1}\right)\right), \tau \Theta^{n}\right) \leq \sum_{n=1}^{N} \tau\left(1+\left\|\beta\left(U^{n-1}\right)\right\|_{L^{2}(\Omega)}\right)\left\|\Theta^{n}\right\|_{L^{2}(\Omega)} \\
& \leq \sum_{n=1}^{N} \tau\left(1+\left\|\beta\left(U^{n-1}\right)\right\|_{L^{2}(\Omega)}\right)\left(\frac{1}{\mu}\left\|U^{n}-U^{n-1}\right\|_{L^{2}(\Omega)}+\left\|\beta\left(U^{n-1}\right)\right\|_{L^{2}(\Omega)}\right) \\
& =\sum_{n=1}^{N} \tau\left\|\beta\left(U^{n-1}\right)\right\|_{L^{2}(\Omega)}+\sum_{n=1}^{N} \tau\left\|\beta\left(U^{n-1}\right)\right\|_{L^{2}(\Omega)}^{2} \\
& +\sum_{n=1}^{N} \tau \frac{1}{\mu}\left\|U^{n}-U^{n-1}\right\|_{L^{2}(\Omega)}+\sum_{n=1}^{N} \tau\left\|\beta\left(U^{n-1}\right)\right\|_{L^{2}(\Omega)}\left(\frac{1}{\mu}\left\|U^{n}-U^{n-1}\right\|_{L^{2}(\Omega)}\right) \\
& \leq \sum_{n=1}^{N}\left(\tau+\tau\left\|\beta\left(U^{n-1}\right)\right\|_{L^{2}(\Omega)}^{2}\right)+\sum_{n=1}^{N} \tau\left\|\beta\left(U^{n-1}\right)\right\|_{L^{2}(\Omega)}^{2} \\
& +\sum_{n=1}^{N}\left(C_{\epsilon} \tau^{2}+\epsilon\left\|U^{n}-U^{n-1}\right\|_{L^{2}(\Omega)}^{2}\right)+\sum_{n=1}^{N}\left(\left\|\tau \beta\left(U^{n-1}\right)\right\|_{L^{2}(\Omega)}^{2}+\epsilon\left\|^{n}-U^{n-1}\right\|_{L^{2}(\Omega)}^{2}\right) \\
& \leq C+\sum_{n=1}^{N} \tau\left\|\beta\left(U^{n-1}\right)\right\|_{L^{2}(\Omega)}^{2}+2 \epsilon \sum_{n=1}^{N}\left\|U^{n}-U^{n-1}\right\|_{L^{2}(\Omega)}^{2} .
\end{aligned}
$$

Putting together these estimates, we get, with $2 \epsilon=\frac{1}{4 \mu}$,

$$
\begin{aligned}
& -C+C\left\|\beta\left(U^{N}\right)\right\|_{L^{2}(\Omega)}^{2}+\frac{1}{2 \mu} \sum_{n=1}^{N}\left\|U^{n}-U^{n-1}\right\|_{L^{2}(\Omega)}^{2}+\tau C \sum_{n=1}^{N}\left\|\Theta^{n}\right\|_{H_{0}^{1}(\Omega)}^{2} \\
& \leq C+C \sum_{n=1}^{N} \tau\left\|\beta\left(U^{n-1}\right)\right\|_{L^{2}(\Omega)}^{2}+\frac{1}{4 \mu} \sum_{n=1}^{N}\left\|U^{n}-U^{n-1}\right\|_{L^{2}(\Omega)}^{2},
\end{aligned}
$$

which implies that

$$
\begin{aligned}
& C\left\|\beta\left(U^{N}\right)\right\|_{L^{2}(\Omega)}^{2}+\frac{1}{4 \mu} \sum_{n=1}^{N}\left\|U^{n}-U^{n-1}\right\|_{L^{2}(\Omega)}^{2}+\tau C \sum_{n=1}^{N}\left\|\beta\left(U^{n}\right)\right\|_{L^{2}(\Omega)}^{2} \\
& \leq C+C \sum_{n=1}^{N} \tau\left\|\beta\left(U^{n-1}\right)\right\|_{L^{2}(\Omega)}^{2} .
\end{aligned}
$$

By Gronwall lemma, we derive

$$
\max _{1 \leq n \leq N}\left\|\beta\left(U^{n}\right)\right\|_{L^{2}(\Omega)}+\sum_{n=1}^{N}\left\|U^{n}-U^{n-1}\right\|_{L^{2}(\Omega)}^{2}+\sum_{n=1}^{N} \tau\left\|\Theta^{n}\right\|_{H^{1}(\Omega)}^{2} \leq C,
$$

and the proof of Lemma 4.1 is now complete.

Proof of Theorem 5.1. Denote

$$
F(\beta(u))=\frac{\lambda f(\beta(u))}{\left(\int_{\Omega} f(\beta(u)) d x\right)^{2}} .
$$


Then (1.1)- (1.3) can be rewriten as

$$
\begin{aligned}
& \frac{\partial u}{\partial t}-\Delta \beta(u)=F(\beta(u)), \quad \text { in } \Omega \times(0, T), \\
& \beta(u)=0, \quad \text { on } \partial \Omega \times(0, T), \\
& u(0)=u_{0} . \quad \text { in } \Omega,
\end{aligned}
$$

The variational form of (7.2) - (7.4) is to find $u \in H_{0}^{1}(\Omega)$ such that, with $\theta=\beta(u)$,

$$
\left(u_{t}, v\right)+(\nabla \theta, \nabla v)=(F(\beta(u)), v), \quad \text { for any } v \in H_{0}^{1}(\Omega),
$$

or integrating on $\left(t_{n-1}, t_{n}\right)$, with $u^{n}=u\left(t_{n}\right)$,

$$
\left(u^{n}-u^{n-1}, v\right)+\tau\left(\nabla \bar{\theta}^{n}, \nabla v\right)=\tau\left(\overline{F(\beta(u)}^{n}, v\right), \quad \text { for any } v \in H_{0}^{1}(\Omega) .
$$

Here

$$
\bar{\theta}^{n}=\frac{1}{\tau} \int_{t_{n-1}}^{t_{n}} \theta(t) d t, \quad \overline{F(\beta(u))}^{n}=\frac{1}{\tau} \int_{t_{n-1}}^{t_{n}} F(\beta(u(t))) d t .
$$

The time discretization scheme (3.10) - (3.12) can be written as

$$
\begin{aligned}
& \left(U^{n}-U^{n-1}, v\right)+\tau\left(\nabla \Theta^{n}, \nabla v\right)=\tau\left(F\left(U^{n-1}\right), v\right), \quad \forall v \in H_{0}^{1}(\Omega), \\
& U^{n}-U^{n-1}=\mu\left(\Theta^{n}-\beta\left(U^{n-1}\right)\right) .
\end{aligned}
$$

Subtracting (7.7) from (7.6) and summing up from 1 to $i$, we obtain

$$
\begin{aligned}
& \sum_{n=1}^{i}\left(\left(u^{n}-U^{n}\right)-\left(u^{n-1}-U^{n-1}\right), v\right)+\tau \sum_{n=1}^{i}\left(\nabla\left(\bar{\theta}^{n}-\Theta^{n}\right), \nabla v\right) \\
& =\tau \sum_{n=1}^{i}\left(\overline{F(\beta(u)}^{n}-F\left(U^{n-1}\right), v\right), \quad \text { for any } v \in H_{0}^{1}(\Omega),
\end{aligned}
$$

or, noting that $U^{0}=u_{0}=u(0)$,

$$
\begin{aligned}
& \left(u^{i}-U^{i}, v\right)+\tau \sum_{n=1}^{i}\left(\nabla\left(\bar{\theta}^{n}-\Theta^{n}\right), \nabla v\right) \\
& =\tau \sum_{n=1}^{i}\left(\overline{F(\beta(u))}^{n}-F\left(U^{n-1}\right), v\right), \quad \text { for any } v \in H_{0}^{1}(\Omega) .
\end{aligned}
$$

Choosing $v=\tau\left(\bar{\theta}^{i}-\Theta^{i}\right)$ in (7.9) and summing up from 1 to $N$, we derive

$$
\begin{aligned}
& \sum_{i=1}^{N}\left(u^{i}-U^{i}, \tau\left(\bar{\theta}^{i}-\Theta^{i}\right)\right)+\tau^{2} \sum_{i=1}^{N} \sum_{n=1}^{i}\left(\nabla\left(\bar{\theta}^{n}-\Theta^{n}\right), \nabla\left(\bar{\theta}^{i}-\Theta^{i}\right)\right) \\
& =\tau \sum_{n=1}^{i}\left(\overline{F(\beta(u)}^{n}-F\left(U^{n-1}\right), \tau\left(\bar{\theta}^{i}-\Theta^{i}\right)\right), \quad \text { for any } v \in H_{0}^{1}(\Omega) .
\end{aligned}
$$

Noting that

$$
\begin{aligned}
& \left(u^{i}-U^{i}, \tau\left(\bar{\theta}^{i}-\Theta^{i}\right)\right)=\int_{t_{i-1}}^{t_{i}}\left(u^{i}-U^{i}, \theta(t)-\Theta^{i}\right) d t \\
& =\int_{t_{i-1}}^{t_{i}}\left(u^{i}-U^{i}, e_{\theta}(t)\right) d t=\int_{t_{i-1}}^{t_{i}}\left(u^{i}-u(t)+u(t)-U^{i}, e_{\theta}(t)\right) d t \\
& =\int_{t_{i-1}}^{t_{i}}\left(e_{u}(t), e_{\theta}(t)\right) d t-\int_{t_{i-1}}^{t_{i}}\left(u(t)-u^{i}, e_{\theta}(t)\right) d t
\end{aligned}
$$


we obtain

$$
\begin{aligned}
& I+I I=\sum_{i=1}^{N} \int_{t_{i-1}}^{t_{i}}\left(e_{u}(t), e_{\theta}(t)\right) d t+\tau^{2} \sum_{i=1}^{N} \sum_{n=1}^{i}\left(\nabla\left(\bar{\theta}^{n}-\Theta^{n}\right), \nabla\left(\bar{\theta}^{i}-\Theta^{i}\right)\right) \\
& =\sum_{i=1}^{N} \int_{t_{i-1}}^{t_{i}}\left(u(t)-u^{i}, e_{\theta}(t)\right) d t+\tau^{2} \sum_{n=1}^{i}\left(\overline{F(\beta(u))^{n}}-F\left(U^{n-1}\right), \bar{\theta}^{i}-\Theta^{i}\right) \\
& =I I I+I V .
\end{aligned}
$$

For $t \in\left(t_{i-1}, t_{i}\right)$, we have

$$
e_{u}(t)=u(t)-U^{i}=\mu \theta(t)+\alpha(u)-U^{i}=\mu e_{\theta}(t)+\alpha(u)-\alpha\left(U^{i-1}\right),
$$

and

$$
e_{\theta}(t)=\beta(u(t))-\Theta^{i}=\beta(u(t))-\beta\left(U^{i-1}\right)-\frac{1}{\mu}\left(U^{i}-U^{i-1}\right) .
$$

Hence

$$
\begin{aligned}
I= & \sum_{i=1}^{N} \int_{t_{i-1}}^{t_{i}}\left(e_{u}(t), e_{\theta}(t)\right) d t \\
= & \sum_{i=1}^{N} \int_{t_{i-1}}^{t_{i}} \mu\left(e_{\theta}(t), e_{\theta}(t)\right) d t+\sum_{i=1}^{N} \int_{t_{i-1}}^{t_{i}}\left(\alpha(u)-\alpha\left(U^{i-1}\right), e_{\theta}(t)\right) d t \\
= & \sum_{i=1}^{N} \int_{t_{i-1}}^{t_{i}} \mu\left\|e_{\theta}(t)\right\|_{L^{2}(\Omega)}^{2} d t+\sum_{i=1}^{N} \int_{t_{i-1}}^{t_{i}}\left(\alpha(u)-\alpha\left(U^{i-1}\right), \beta(u)-\beta\left(U^{i-1}\right)\right) d t \\
& \quad+\sum_{i=1}^{N} \int_{t_{i-1}}^{t_{i}}\left(\alpha(u)-\alpha\left(U^{i-1}\right),-\frac{1}{\mu}\left(U^{i}-U^{i-1}\right)\right) d t .
\end{aligned}
$$

Furthermore, we have

$$
\alpha(u)-\alpha\left(U^{i-1}\right)=u(t)-\mu \theta(t)-U^{i-1}+\mu \beta\left(U^{i-1}\right)=u(t)-U^{i}-\mu e_{\theta}(t) .
$$

Thus

$$
\begin{aligned}
I= & \mu \sum_{i=1}^{N} \int_{t_{i-1}}^{t_{i}}\left\|e_{\theta}(t)\right\|_{L^{2}(\Omega)}^{2} d t+\sum_{i=1}^{N} \int_{t_{i-1}}^{t_{i}}\left(\alpha(u)-\alpha\left(U^{i-1}\right), \beta(u)-\beta\left(U^{i-1}\right)\right) d t \\
& +\frac{\tau}{\mu} \sum_{i=1}^{N}\left(U^{i},\left(U^{i}-U^{i-1}\right)\right)+\sum_{i=1}^{N} \int_{t_{i-1}}^{t_{i}}\left(e_{\theta}(t),\left(U^{i}-U^{i-1}\right)\right) d t \\
& -\frac{1}{\mu} \sum_{i=1}^{N} \int_{t_{i-1}}^{t_{i}}\left(u(t),\left(U^{i}-U^{i-1}\right)\right) d t=I_{1}^{N}+I_{2}+I_{3}+I_{4}+I_{5} .
\end{aligned}
$$

For $I_{2}$, we have, since $\alpha, \beta$ are increasing,

$$
I_{2}=\sum_{i=1}^{N} \int_{t_{i-1}}^{t_{i}}\left(\alpha(u)-\alpha\left(U^{i-1}\right), \beta(u)-\beta\left(U^{i-1}\right)\right) d t \geq 0 .
$$


For $I_{3}$, we have, using the equality $2 a(a-b)=a^{2}-b^{2}+(a-b)^{2}$,

$$
\begin{aligned}
I_{3} & =\frac{\tau}{\mu} \sum_{i=1}^{N}\left(U^{i}, U^{i}-U^{i-1}\right) \\
& =\frac{\tau}{\mu} \sum_{i=1}^{N}\left(\left\|U^{i}\right\|_{L^{2}(\Omega)}^{2}-\left\|U^{i-1}\right\|_{L^{2}(\Omega)}^{2}+\left\|U^{i}-U^{i-1}\right\|_{L^{2}(\Omega)}^{2}\right) \\
& \geq \frac{\tau}{2 \mu} \sum_{i=1}^{N}\left(\left\|U^{i}\right\|_{L^{2}(\Omega)}^{2}-\left\|U^{i-1}\right\|_{L^{2}(\Omega)}^{2}\right) \\
& \geq \frac{\tau}{2 \mu}\left(\left\|U^{N}\right\|_{L^{2}(\Omega)}^{2}-\left\|U^{0}\right\|_{L^{2}(\Omega)}^{2}\right) \geq-\frac{\tau}{2 \mu}\left\|U^{0}\right\|_{L^{2}(\Omega)}^{2} .
\end{aligned}
$$

For $I_{4}$, we have, by Young inequality $a b \leq \epsilon a^{2}+C_{\epsilon} b^{2}$,

$$
\begin{aligned}
\left|I_{4}\right| & \left.=\left|\sum_{i=1}^{N} \int_{t_{i-1}}^{t_{i}}\left(e_{\theta}(t), U^{i}-U^{i-1}\right) d t\right| \leq \sum_{i=1}^{N} \int_{t_{i-1}}^{t_{i}}\left\|e_{\theta}(t)\right\|_{L^{2}(\Omega)} \| U^{i}-U^{i-1}\right) \|_{L^{2}(\Omega)} d t \\
& \leq \epsilon \sum_{i=1}^{N} \int_{t_{i-1}}^{t_{i}}\left\|e_{\theta}\right\|_{L^{2}(\Omega)}^{2} d t+C_{\epsilon} \sum_{i=1}^{N} \tau\left\|U^{i}-U^{i-1}\right\|_{L^{2}(\Omega)}^{2} \\
& \leq \frac{1}{4} I_{1}^{N}+C \tau
\end{aligned}
$$

where the last inequality follows from Lemma 4.1. Thus we derive

$$
I \geq I_{1}^{N}-\frac{\tau}{2 \mu}\left\|U^{0}\right\|_{L^{2}(\Omega)}^{2}-\left|I_{4}\right|+I_{5}
$$

For II, we have, using the equality $2 \sum_{i=1}^{N} a_{i}\left(\sum_{n=1}^{i} a_{n}\right)=\left(\sum_{i=1}^{N} a_{i}\right)^{2}+\sum_{i=1}^{N} a_{i}^{2}$,

$$
\begin{aligned}
I I & =\tau^{2} \sum_{i=1}^{N} \sum_{n=1}^{i}\left(\nabla\left(\bar{\theta}^{i}-\Theta^{i}\right), \nabla\left(\bar{\theta}^{n}-\Theta^{n}\right)\right) \geq \frac{1}{2} \tau^{2}\left\|\sum_{i=1}^{N} \nabla\left(\bar{\theta}^{n}-\Theta^{n}\right)\right\|_{L^{2}(\Omega)}^{2} \\
& =\frac{1}{2}\left\|\sum_{i=1}^{N} \int_{t_{i-1}}^{t_{i}} \nabla\left(\theta(t)-\Theta^{i}\right) d t\right\|_{L^{2}(\Omega)}^{2}=\frac{1}{2}\left\|\nabla \int_{t_{0}}^{t_{N}} e_{\theta}(t) d t\right\|_{L^{2}(\Omega)}^{2}
\end{aligned}
$$

For III, we have

$$
\begin{aligned}
I I I & =\left|\sum_{i=1}^{N} \int_{t_{i-1}}^{t_{i}}\left(u(t)-u^{i}, e_{\theta}(t)\right) d t\right|=\left|\sum_{i=1}^{N} \int_{t_{i-1}}^{t_{i}}\left(-\int_{t}^{t_{i}} \frac{\partial u}{\partial s} d s, e_{\theta}(t)\right) d t\right| \\
& \left.\leq \sum_{i=1}^{N} \int_{t_{i-1}}^{t_{i}} \int_{t_{i-1}}^{t_{i}}\left\|\frac{\partial u}{\partial s}\right\|_{H^{-1}} d s\left\|e_{\theta}(t)\right\|_{H^{1}(\Omega)}\right) d t \\
& \leq\left[\sum_{i=1}^{N}\left(\int_{t_{i-1}}^{t_{i}}\left\|\frac{\partial u}{\partial s}\right\|_{H^{-1}(\Omega)} d s\right)^{2}\right]^{\frac{1}{2}}\left[\sum_{i=1}^{N}\left(\int_{t_{i-1}}^{t_{i}}\left\|e_{\theta}(t)\right\|_{H^{1}(\Omega)} d t\right)^{2}\right]^{\frac{1}{2}} \\
& \leq \tau\left(\int_{0}^{t_{N}}\left\|\frac{\partial u}{\partial s}\right\|_{H^{-1}(\Omega)}^{2} d s\right)^{\frac{1}{2}}\left(\int_{0}^{t_{N}}\left\|e_{\theta}(t)\right\|_{H^{1}(\Omega)}^{2} d t\right)^{\frac{1}{2}} .
\end{aligned}
$$


For IV, we have

$$
\begin{aligned}
I V & =\left|\sum_{i=1}^{N} \int_{t_{i-1}}^{t_{i}}\left(\sum_{n=1}^{i} \tau\left[\overline{F(\beta(u))^{n}}-F\left(\beta\left(U^{n-1}\right)\right)\right], \bar{\theta}^{i}-\Theta^{i}\right) d t\right| \\
& =\left|\sum_{i=1}^{N} \int_{t_{i-1}}^{t_{i}}\left(\sum_{n=1}^{i} \tau\left[\overline{F(\beta(u)}^{n}-F\left(\beta\left(U^{n-1}\right)\right)\right], e_{\theta}(t)\right) d t\right| \\
& \leq \epsilon \int_{0}^{t_{N}}\left\|e_{\theta}(t)\right\|_{L^{2}(\Omega)}^{2} d t+C_{\epsilon} \sum_{i=1}^{N} \tau\left\|\sum_{n=1}^{i} \tau\left[\overline{F(\beta(u))}^{n}-F\left(\beta\left(U^{n-1}\right)\right)\right]\right\|_{L^{2}(\Omega)}^{2} \\
& \leq \epsilon \int_{0}^{t_{N}}\left\|e_{\theta}(t)\right\|_{L^{2}(\Omega)}^{2} d t+C_{\epsilon} \sum_{i=1}^{N} \tau\left(\sum_{n=1}^{i} \tau\left\|\overline{F(\beta(u))^{n}}-F\left(\beta\left(U^{n-1}\right)\right)\right\|_{L^{2}(\Omega)}^{2}\right) \\
& =\epsilon \int_{0}^{t_{N}}\left\|e_{\theta}(t)\right\|_{L^{2}(\Omega)}^{2} d t+C_{\epsilon} \sum_{i=1}^{N} \tau\left(\sum_{n=1}^{i} \tau\left\|\frac{1}{\tau} \int_{t_{n-1}}^{t_{n}}\left[F(\beta(u))-F\left(\beta\left(U^{n-1}\right)\right)\right] d s\right\|_{L^{2}(\Omega)}^{2}\right) \\
& =\epsilon \int_{0}^{t_{N}}\left\|e_{\theta}(t)\right\|_{L^{2}(\Omega)}^{2} d t+C_{\epsilon} \sum_{i=1}^{N} \tau\left(\sum_{n=1}^{i} \int_{t_{n-1}}^{t_{n}}\left\|F(\beta(u))-F\left(\beta\left(U^{n-1}\right)\right)\right\|_{L^{2}(\Omega)}^{2} d s\right) .
\end{aligned}
$$

Since $\beta$ and $f$ satisfy Lipschitz conditions, we have, noting that $\left\|U^{n}\right\|_{L^{2}(\Omega)} \leq C,\left\|f\left(\beta\left(U^{n-1}\right)\right)\right\|_{L^{2}(\Omega)} \leq C$ and $\|f(\beta(u))\|_{L^{2}(\Omega)} \leq C$ and $\beta(u) \geq \sigma>0$,

$$
\begin{aligned}
& \left|F(\beta(u))-F\left(\beta\left(U^{n-1}\right)\right)\right|=\left|\frac{\lambda f(\beta(u))}{\left(\int_{\Omega} f(\beta(u)) d x\right)^{2}}-\frac{\lambda f\left(\beta\left(U^{n-1}\right)\right)}{\left(\int_{\Omega} f\left(\beta\left(U^{n-1}\right)\right) d x\right)^{2}}\right| \\
& \leq C\left|\frac{f(\beta(u))-f\left(\beta\left(U^{n-1}\right)\right)}{\left(\int_{\Omega} f(\beta(u)) d x\right)^{2}}\right| \\
& +\left|\frac{f\left(\beta\left(U^{n-1}\right)\right)\left[\int_{\Omega} f(\beta(u))-f\left(\beta\left(U^{n-1}\right)\right) d x\right]\left[\int_{\Omega} f(\beta(u))+f\left(\beta\left(U^{n-1}\right)\right) d x\right]}{\left(\int_{\Omega} f(\beta(u)) d x\right)^{2}\left(\int_{\Omega} f\left(\beta\left(U^{n-1}\right)\right) d x\right)^{2}}\right| \\
& \leq C\left|\beta(u)-\beta\left(U^{n-1}\right)\right|+C \int_{\Omega}\left|f(\beta(u))-f\left(\beta\left(U^{n-1}\right)\right)\right| d x \\
& \leq C\left|\beta(u)-\beta\left(U^{n-1}\right)\right|+C\left(\int_{\Omega}\left|f(\beta(u))-f\left(\beta\left(U^{n-1}\right)\right)\right|^{2} d x\right)^{\frac{1}{2}} \\
& \leq C\left|\beta(u)-\beta\left(U^{n-1}\right)\right|+C\left\|\beta(u)-\beta\left(U^{n-1}\right)\right\|_{L^{2}(\Omega)} .
\end{aligned}
$$

Further we have

$$
\beta(u)-\beta\left(U^{n-1}\right)=e_{\theta}(t)+\frac{1}{\mu}\left(U^{n}-U^{n-1}\right) .
$$

Thus

$$
\begin{aligned}
& \left\|F(\beta(u))-F\left(\beta\left(U^{n-1}\right)\right)\right\|_{L^{2}(\Omega)}^{2}=\int_{\Omega}\left|F(\beta(u))-F\left(\beta\left(U^{n-1}\right)\right)\right|^{2} d x \\
& \leq C \int_{\Omega}\left|\beta(u)-\beta\left(U^{n-1}\right)\right|^{2} d x+C\left\|\beta(u)-\beta\left(U^{n-1}\right)\right\|_{L^{2}(\Omega)}^{2} \\
& \leq C\left\|\beta(u)-\beta\left(U^{n-1}\right)\right\|_{L^{2}(\Omega)}^{2} \leq C\left\|e_{\theta}(t)\right\|_{L^{2}(\Omega)}^{2}+C\left\|U^{n}-U^{n-1}\right\|_{L^{2}(\Omega)}^{2},
\end{aligned}
$$


which implies that

$$
\begin{aligned}
I V \leq \epsilon & \int_{0}^{T_{N}}\left\|e_{\theta}(t)\right\|_{L^{2}(\Omega)}^{2} d t \\
& \quad+C_{\epsilon} \sum_{i=1}^{N} \tau\left[\sum_{n=1}^{i} \int_{t_{n-1}}^{t_{n}}\left(\left\|e_{\theta}(t)\right\|_{L^{2}(\Omega)}^{2}+\left\|U^{n}-U^{n-1}\right\|_{L^{2}(\Omega)}^{2}\right) d t\right] \\
= & \frac{1}{4} \int_{0}^{T_{N}}\left\|e_{\theta}(t)\right\|_{L^{2}(\Omega)}^{2} d t+C_{\epsilon} \sum_{i=1}^{N} \tau\left[\sum_{n=1}^{i} \int_{t_{n-1}}^{t_{n}}\left\|e_{\theta}(t)\right\|_{L^{2}(\Omega)}^{2} d t\right] \\
& \quad+C_{\epsilon} \sum_{i=1}^{N} \tau\left[\sum_{n=1}^{i} \int_{t_{n-1}}^{t_{n}}\left\|U^{n}-U^{n-1}\right\|_{L^{2}(\Omega)}^{2} d t\right] \\
\leq & \frac{1}{4} I_{1}^{N}+C \sum_{i=1}^{N} \tau I_{1}^{i}+C \sum_{i=1}^{N} \tau\left[\sum_{n=1}^{i} \tau\left\|U^{n}-U^{n-1}\right\|_{L^{2}(\Omega)}^{2}\right] \\
= & \frac{1}{4} I_{1}^{N}+C \sum_{i=1}^{N} \tau I_{1}^{i}+C \sum_{i=1}^{N} \tau\left\|U^{i}-U^{i-1}\right\|_{L^{2}(\Omega)}^{2} \\
\leq & \frac{1}{4} I_{1}^{N}+C \sum_{i=1}^{N} \tau I_{1}^{i}+C \tau .
\end{aligned}
$$

Putting together these estimates we obtain

$$
\begin{aligned}
& I_{1}^{N}-C \tau-\left|I_{4}\right|+I_{5}+\frac{1}{4}\left\|\nabla \int_{0}^{T_{N}} e_{\theta}(t) d t\right\|_{L^{2}(\Omega)}^{2} \\
& \leq C \tau+\frac{1}{4} I_{1}^{N}+C \sum_{i=1}^{N} \tau I_{1}^{i}+C \tau .
\end{aligned}
$$

Further we note that $\left|I_{4}\right| \leq \frac{1}{4} I_{1}^{N}$, we therefore obtain

$$
\begin{aligned}
& \frac{\mu}{4}\left\|e_{\theta}\right\|_{L_{2}(\Omega \times(0, T))}^{2}+\frac{1}{4}\left\|\nabla \int_{0}^{T_{N}} e_{\theta}(t) d t\right\|_{L^{2}(\Omega)}^{2} \\
& \leq C \sum_{i=1}^{N} \tau\left\|e_{\theta}\right\|_{L^{2}\left(0, t_{i} ; L^{2}(\Omega)\right)}^{2}+C \tau+\frac{1}{\mu} \sum_{i=1}^{N} \int_{t_{i-1}}^{t_{i}}\left(u(t), U^{i}-U^{i-1}\right) d t .
\end{aligned}
$$

By Lemma 5.2 and Gronwall lemma, we have

$$
\left\|e_{\theta}\right\|_{L^{2}(\Omega \times(0, T))}+\left\|\int_{0}^{t} e_{\theta}\right\|_{L^{\infty}\left(0, T ; H^{1}(\Omega)\right)}=O\left(\tau^{\frac{1}{2}}\right) .
$$

These estimates lead to the following $H^{-1}(\Omega)$-error bound for the unknown $u$ (see (4.16) in [16]),

$$
\left\|e_{u}\right\|_{L^{\infty}\left(0, T ; H^{-1}(\Omega)\right)} \leq C \tau^{\frac{1}{2}}
$$

Together these estimates complete the proof of Theorem 5.1. 